\title{
Hemichordate genomes and deuterostome origins
}

Oleg Simakov ${ }^{1,2 *}$, Takeshi Kawashima ${ }^{3}+*$, Ferdinand Marlétaz ${ }^{4}$, Jerry Jenkins ${ }^{5}$, Ryo Koyanagi ${ }^{6}$, Therese Mitros ${ }^{7}$, Kanako Hisata $^{3}$, Jessen Bredeson ${ }^{7}$, Eiichi Shoguchi ${ }^{3}$, Fuki Gyoja ${ }^{3}$, Jia-Xing Yue ${ }^{8}+$, Yi-Chih Chen ${ }^{9}$, Robert M. Freeman Jr ${ }^{10}+$, Akane Sasaki ${ }^{11}$, Tomoe Hikosaka-Katayama ${ }^{12}$, Atsuko Sato ${ }^{13}$, Manabu Fujie ${ }^{6}$, Kenneth W. Baughman ${ }^{3}$, Judith Levine ${ }^{14}$, Paul Gonzalez ${ }^{14}$, Christopher Cameron ${ }^{15}$, Jens H. Fritzenwanker ${ }^{14}$, Ariel M. Pani ${ }^{16}$, Hiroki Goto ${ }^{6}$, Miyuki Kanda ${ }^{6}$, Nana Arakaki ${ }^{6}$, Shinichi Yamasaki ${ }^{6}$, Jiaxin $\mathrm{Qu}^{17}$, Andrew Cree ${ }^{17}$, Yan Ding ${ }^{17}$, Huyen H. Dinh ${ }^{17}$, Shannon Dugan ${ }^{17}$, Michael Holder ${ }^{17}$, Shalini N. Jhangiani1 ${ }^{17}$, Christie L. Kovar ${ }^{17}$, Sandra L. Lee ${ }^{17}$, Lora R. Lewis ${ }^{17}$, Donna Morton ${ }^{17}$, Lynne V. Nazareth ${ }^{17}$, Geoffrey Okwuonu ${ }^{17}$, Jireh Santibanez ${ }^{17}$, Rui Chen ${ }^{17}$, Stephen Richards ${ }^{17}$, Donna M. Muzny ${ }^{17}$, Andrew Gillis ${ }^{18}$, Leonid Peshkin ${ }^{10}$,

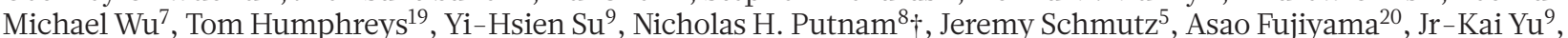
Kunifumi Tagawa ${ }^{11}$, Kim C. Worley ${ }^{17}$, Richard A. Gibbs ${ }^{17}$, Marc W. Kirschner ${ }^{10}$, Christopher J. Lowe ${ }^{14}$, Noriyuki Satoh ${ }^{3}$, Daniel S. Rokhsar ${ }^{1,7,21} \&$ John Gerhart ${ }^{7}$

Acorn worms, also known as enteropneust (literally, 'gut-breathing') hemichordates, are marine invertebrates that share features with echinoderms and chordates. Together, these three phyla comprise the deuterostomes. Here we report the draft genome sequences of two acorn worms, Saccoglossus kowalevskii and Ptychodera flava. By comparing them with diverse bilaterian genomes, we identify shared traits that were probably inherited from the last common deuterostome ancestor, and then explore evolutionary trajectories leading from this ancestor to hemichordates, echinoderms and chordates. The hemichordate genomes exhibit extensive conserved synteny with amphioxus and other bilaterians, and deeply conserved non-coding sequences that are candidates for conserved gene-regulatory elements. Notably, hemichordates possess a deuterostome-specific genomic cluster of four ordered transcription factor genes, the expression of which is associated with the development of pharyngeal 'gill' slits, the foremost morphological innovation of early deuterostomes, and is probably central to their filter-feeding lifestyle. Comparative analysis reveals numerous deuterostome-specific gene novelties, including genes found in deuterostomes and marine microbes, but not other animals. The putative functions of these genes can be linked to physiological, metabolic and developmental specializations of the filter-feeding ancestor.

The prominent pharyngeal gill slits, rigid stomochord, and midline nerve cords of acorn worms led 19th century zoologists to designate them as 'hemichordates' and group them with vertebrates and other chordates $^{1-4}$, but their early embryos and larvae also linked them to echinoderms ${ }^{5,6}$. Current molecular phylogenies strongly support the affinities of hemichordates and echinoderms as sister phyla, together called ambulacrarians ${ }^{7}$, and unite ambulacrarians and chordates within the deuterostomes (see glossary in Supplementary Note 1). Of all the shared derived morphological characters proposed between hemichordates and chordates, the pharyngeal gill slits have emerged with unambiguous morphological and molecular support, notably the shared expression of the pax $1 / 9$ gene $^{8-10}$. These structures were ancestral deuterostome characters elaborated upon the bilaterian ancestral body plan, but the gill slits were subsequently lost in extant echinoderms and amniotes ${ }^{11}$. Since extant invertebrate deuterostomes use this apparatus for efficient suspension and/or deposit feeding, the early Cambrian or Precambrian deuterostome ancestor probably also shared this lifestyle. This perspective on the last common deuterostome ancestor informs our understanding of the subsequent evolution of hemichordates, echinoderms and chordates ${ }^{10,12-16}$.

Hemichordates share bilateral symmetry, gill slits, soft bodies and early axial patterning with chordates, making them key comparators for inferring the ancestral genomic features of deuterostomes. To this end, we sequenced and analysed the genomes of acorn worms belonging to the two main lineages of enteropneust hemichordates (Supplementary Note 1): Saccoglossus kowalevskii (Harrimaniidae; Atlantic, North America, Fig. 1a) and Ptychodera flava (Ptychoderidae; Pacific, pan-tropical, Fig. 1b). Both have characteristic three-part

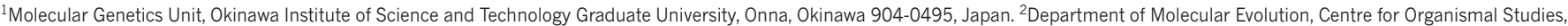
University of Heidelberg, 69115 Heidelberg, Germany. ${ }^{3}$ Marine Genomics Unit, Okinawa Institute of Science and Technology Graduate University, Onna, Okinawa 904-0495, Japan. ${ }^{4}$ Department of Zoology, University of Oxford, Oxford OX1 3PS, UK. ${ }^{5}$ HudsonAlpha Institute of Biotechnology, Huntsville, Alabama 35806, USA. ${ }^{6}$ DNA Sequencing Section, Okinawa Institute of Science and Technology Graduate University, Onna, Okinawa 904-0495, Japan. ${ }^{7}$ Department of Molecular and Cell Biology, University of California, Berkeley California 94720-3200, USA. ${ }^{8}$ Department of Ecology and Evolutionary Biology, Rice University, Houston, Texas 77005, USA. ${ }^{9}$ Institute of Cellular and Organismic Biology, Academia Sinica, Taipei 11529 , Taiwan. ${ }^{10}$ Department of Systems Biology, Harvard Medical School, Boston, Massachusetts 02115, USA. ${ }^{11}$ Marine Biological Laboratory, Graduate School of Science, Hiroshima University, Onomichi, Hiroshima 722-0073, Japan. ${ }^{12}$ Natural Science Center for Basic Research and Development, Gene Science Division, Hiroshima University, Higashi-Hiroshima, Hiroshima 739-8527, Japan. ${ }^{13}$ Marine Biological Association of

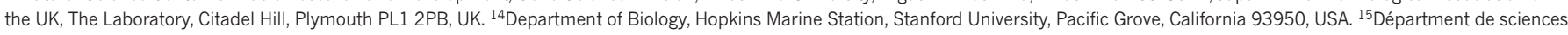
biologiques, University of Montreal, Quebec H3C 3J7. Canada. ${ }^{16}$ University of North Caroline at Chapel Hill, North Carolina 27599, USA. ${ }^{17}$ Human Genome Sequencing Center, Department of

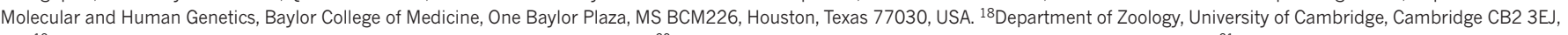

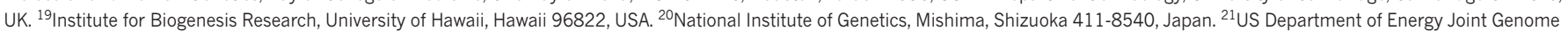

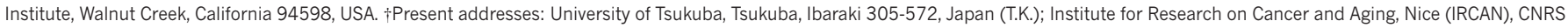

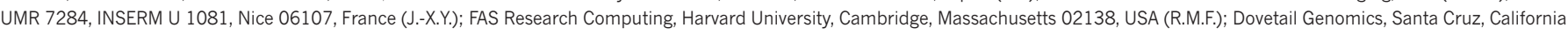
95060, USA (N.H.P.)

*These authors contributed equally to this work.
} 

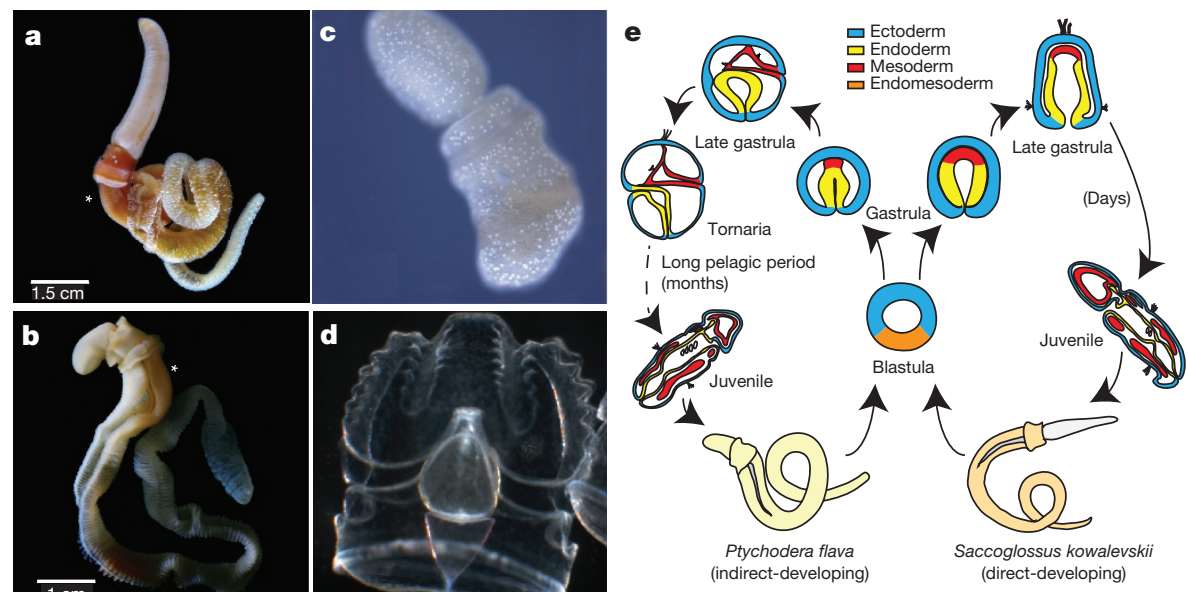

Figure 1 | Hemichordate model systems and their embryonic development. The hemichordate phylum includes the enteropneusts (acorn worms) and pterobranchs (minute, colonial, tube-dwelling; not shown). a, c, Saccoglossus kowalevskii (Harrimaniid (direct developing) enteropneust) adult (a) and juvenile (c) with gill slits. b, d, Ptychodera

bodies comprising proboscis, collar and trunk, the last with tens to hundreds of pairs of gill slits. While S. kowalevskii develops directly to a juvenile worm with these traits within days (Fig. 1c, e), P. flava develops indirectly through a feeding larva that metamorphoses to a juvenile worm after months in the plankton (Fig. 1d, e). Our analyses begin to integrate macroscopic information about morphology, organismal physiology, and descriptive embryology of these deuterostomes with genomic information about gene homologies, gene arrangements, gene novelties and non-coding elements.

\section{Genomes}

We sequenced the two acorn worm genomes by random shotgun methods with a variety of read types (Methods; Supplementary Note 2), each starting from sperm from a single outbred diploid individual. The haploid lengths of the two genomes are both about $1 \mathrm{Gbp}$ (Extended Data Fig. 1), but differ in nucleotide heterozygosity. Both acorn worm genomes were annotated using extensive transcriptome data as well as standard homology-based and de novo methods (Supplementary Note 3). Counting gene models with at least one detectable orthologue in another sequenced metazoan species, we find that Ptychodera and Saccoglossus encode at least 18,556 and 19,270 genes, respectively (Methods). Additional de novo gene predictions include divergent and/ or novel genes (Extended Data Fig. 1). Despite the ancient divergence of the Saccoglossus and Ptychodera lineages (more than 370 million years ago, see below) and their different modes of development, the two acorn worm genomes have similar bulk gene content, as discussed later (Extended Data Fig. 2 and Supplementary Note 4), and similar repetitive landscapes (Supplementary Note 5).

\section{Deuterostome phylogeny}

Deuterostome relationships were originally inferred from developmental and morphological characters ${ }^{2,5,17}$ and these hypotheses were later tested and refined with molecular data ${ }^{6,7}$. Aspects of deuterostome phylogeny continue to be controversial, however, notably the position of the sessile pterobranchs among hemichordates, and the surprising association of Xenoturbella ${ }^{18}$ and acoelomorph flatworms with ambulacrarians ${ }^{19}$ proposed by some studies. We explored these issues using genome-wide analyses of the newly sequenced hemichordate genomes augmented with extensive new RNAseq from five echinoderms, three additional hemichordates (including a rhabdopleurid pterobranch) and two acoels (Fig. 2, Extended Data Fig. 3, Methods and Supplementary Note 6). We recovered the monophyly of hemichordates, echinoderms, ambulacrarians and deuterostomes, using not only amino acid

flava (Ptychoderid (indirect developing) enteropneust) adult (b) and the tornaria stage larva (d). Gill slits labelled with an asterisk in $\mathbf{a}$ and $\mathbf{b}$. e, Comparison of the direct and indirect modes of development of the two hemichordates, indicating the long pelagic larval period in Ptychodera until the settlement and metamorphosis as a juvenile.

characters but also presence-absence characters for introns and coding indels (Supplementary Note 4). Our analyses also placed pterobranch hemichordates as the sister-group to enteropneusts ${ }^{7}$ rather than within them ${ }^{12}$. These phylogenetic analyses imply that genomic traits shared by chordates and ambulacrarians can be attributed to the last common deuterostome ancestor (see below). Using a relaxed molecular clock, we estimate a Cambrian origin of hemichordates (Methods, Extended Data Fig. 3 and Supplementary Note 6).

We also performed several analyses to assess the controversial relationships between Xenoturbella, acoelomorphs and deuterostomes (Supplementary Note 6). With conventional site-homogeneous models, acoels remain outside deuterostomes ${ }^{20-23}$ (Fig. 2, Supplementary Figs 6.1 and 6.2). Alternative models ${ }^{24}$, however, show equivocal branching of acoels depending on the inclusion of the current sparse data for Xenoturbella (Supplementary Note 6). Notably, without Xenoturbella, acoels are positioned as a bilaterian sister group

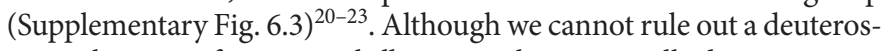
tome placement for Xenoturbella, our analyses generally do not support a grouping of acoels with deuterostomes ${ }^{19}$.

\section{The gene set of the deuterostome ancestor}

By comparative analysis, we identified 8,716 families of homologous genes whose distributions in sequenced extant genomes imply their presence in the deuterostome ancestor (Methods; Supplementary Note 4). Owing to gene duplication and other processes the descendants of these ancestral genes account for $\sim 14,000$ genes in extant deuterostome genomes including human (Supplementary Table 4.1.2). The distributions of gene functions, domain compositions, and gene family sizes of hemichordates resemble those of amphioxus, sea urchin, and sequenced lophotrochozoans more than those of ecdysozoans; vertebrates also form a distinct group (Extended Data Fig. 2, Supplementary Note 4 and Supplementary Fig. 4.2).

Exon-intron structures of genes are generally well conserved among hemichordates, chordates, and many non-deuterostome metazoans, allowing us to infer 2,061 ancestral deuterostome splice sites (Supplementary Note 4). Among orthologous bilaterian genes we found 23 introns and 4 coding sequence indels present only in deuterostomes (shared between at least one ambulacrarian and chordate), suggesting that these shared derived characters may be useful to diagnose clade membership of new candidate organisms (Supplementary Note 4).

Based on whole-genome alignments, we identified 6,533 conserved non-coding elements (CNE) longer than $50 \mathrm{bp}$ that are found in all of the five deuterostomes Saccoglossus, Ptychodera, amphioxus, 


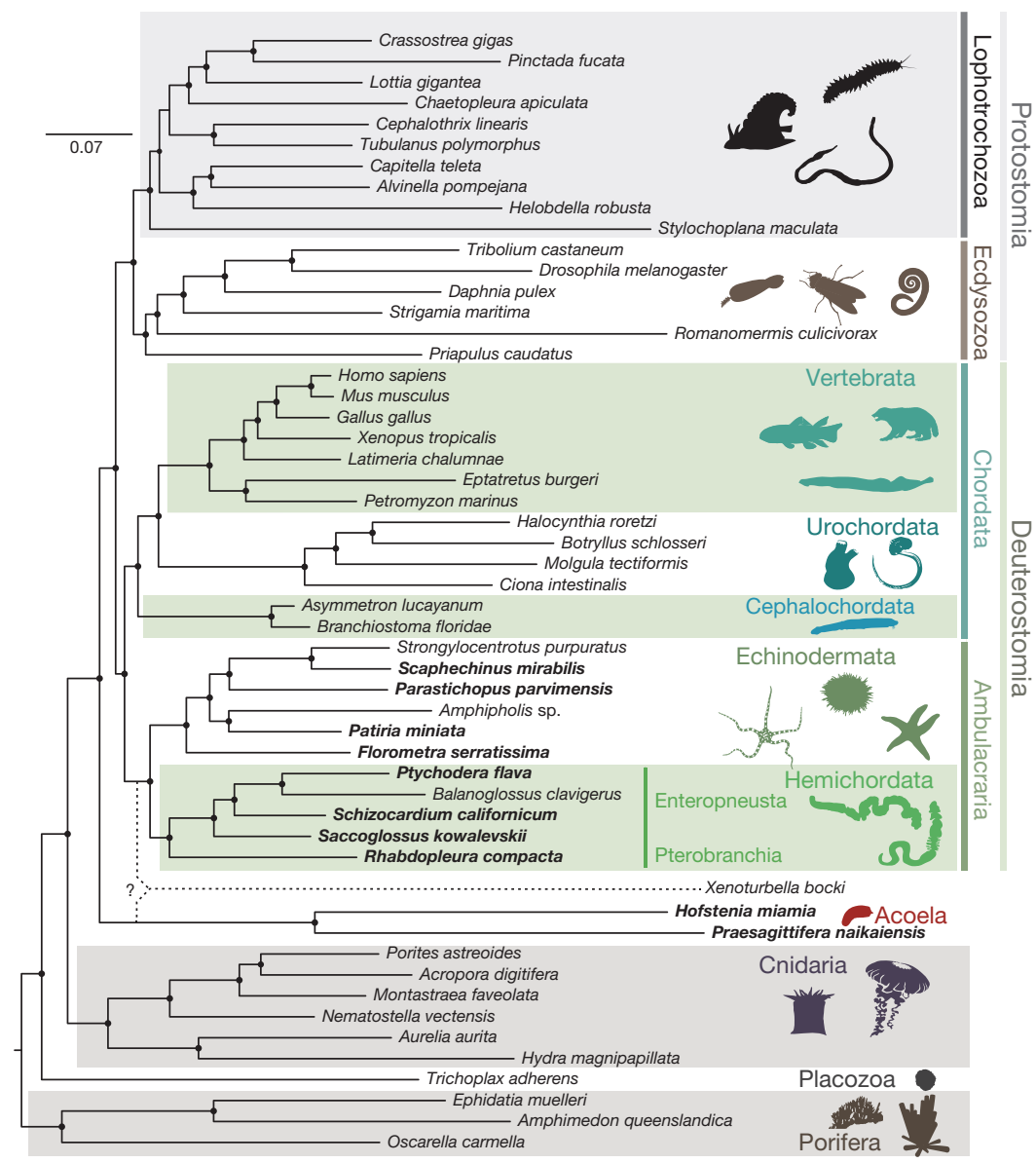

Figure 2 | Phylogenetic placement of deuterostome taxa within the metazoan tree. Maximum-likelihood tree obtained with a super-matrix of 506,428 amino-acid residues gathered from 1,564 orthologous genes in 52 species ( $65.1 \%$ occupancy) and using a $L G+\Gamma$ model partitioned for each

sea urchin, and human (Methods; Supplementary Note 8). The identified CNEs overlap extensively with human long non-coding RNAs (3,611 CNE loci; 55\%, Fisher's exact test $P$ value $\left.<2.2 \times 10^{-16}\right)$. Those alignments usually do not exceed $250 \mathrm{bp}$ (as has been reported among vertebrates ${ }^{25}$ ) and occur in clusters (Supplementary Note 8). Among these conserved sequences is a previously identified vertebrate brain and neural tube specific enhancer, located close to the sox14/21 orthologue in all five species ${ }^{26}$.

\section{Conserved gene linkage}

Ancient gene linkages ('macro-synteny ${ }^{27}$ ) are often preserved in extant bilaterian genomes ${ }^{27,28}$. Comparative analysis revealed 17 ancestral linkage groups across chordates, including amphioxus and Ciona ${ }^{27}$. While the contiguity of the draft of the sea urchin genome assembly ${ }^{29}$ is too limited to determine whether it shares this chromosome-scale organization, we find that the Saccoglossus genome clearly shares these chordate-defined linkage groups (Fig. 3a and Supplementary Note 7), implying that these chromosome-scale linkages were also present in the ancestral deuterostome.

On a more local scale, we find hundreds of tightly linked conserved gene clusters of three or more genes ('micro-synteny'; Methods; Supplementary Note 7) including $\operatorname{Hox}^{30}$ and ParaHox ${ }^{31}$ clusters in both acorn worms (Extended Data Fig. 4), as also found in echinoderms ${ }^{32,33}$. Saccoglossus and amphioxus share more micro-syntenic linkages with each other than either does with sea urchin, vertebrates, or available protostome genomes (Methods, Fig. 3b and Extended Data Figs 5 and 6). Conservation of micro-syntenic linkages can occur due to low rates of genomic rearrangement or, more interestingly, as a result of selection gene. Filled circles at nodes denote maximal bootstrap support. Taxa highlighted in bold are newly sequenced genomes and transcriptomes introduced in this study. Bar indicates the number of substitutions per site.

to retain linkages between genes and their regulatory elements located in neighbouring genes ${ }^{28}$.

\section{A deuterostome pharyngeal gene cluster}

One conserved deuterostome-specific micro-syntenic cluster with functional implications for deuterostome biology is a cluster of genes expressed in the pharyngeal slits and surrounding pharyngeal endoderm (Fig. 4; Supplementary Note 9). This six-gene cluster contains four transcription factor genes in the order $n k \times 2.1, n k x 2.2, \operatorname{pax} 1 / 9$ and foxA, along with two non-transcription-factor genes slc25A21 and mipol1, whose introns harbour regulatory elements for pax 1/9 and fox $A$, respectively ${ }^{34-36}$. The cluster was first found conserved across vertebrates including humans (see chromosome 14; $1.1 \mathrm{Mb}$ length from $n k x 2.1$ to foxA1 $)^{34,37}$. In S. kowalevskii, it is intact with the same gene order as in vertebrates ( $0.5 \mathrm{Mb}$ length from $n k x 2.1$ to foxA), implying that it was present in the deuterostome and ambulacrarian ancestors. The full ordered gene cluster also exists on a single scaffold in the crown-of-thorns sea star Acanthaster planci. Since these genes are not clustered in available protostome genomes, there is no evidence for deeper bilaterian ancestry. Two non-coding elements that are conserved across vertebrates and amphioxus ${ }^{38}$ are found in the hemichordate and A. planci clusters at similar locations (A2 and A4, in Fig. 4a).

The pax1/9 gene, at the centre of the cluster, is expressed in the pharyngeal endodermal primordium of the gill slit in hemichordates, tunicates, amphioxus, fish, and amphibians ${ }^{8,9}$, and in the branchial pouch endoderm of amniotes (which do not complete the last steps of gill slit formation), as well as other locations in vertebrates. The $n k x 2.1$ (thyroid transcription factor 1 ) gene is also expressed in the hemichordate 


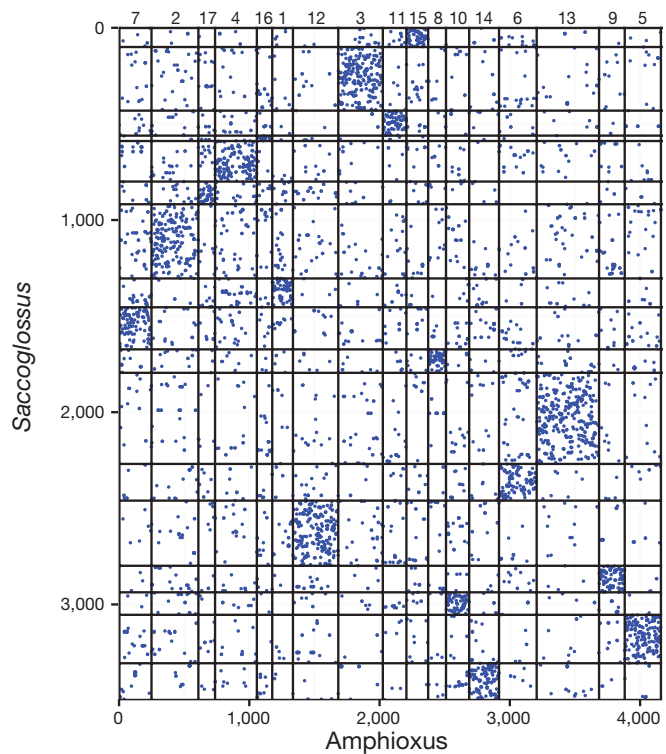

b

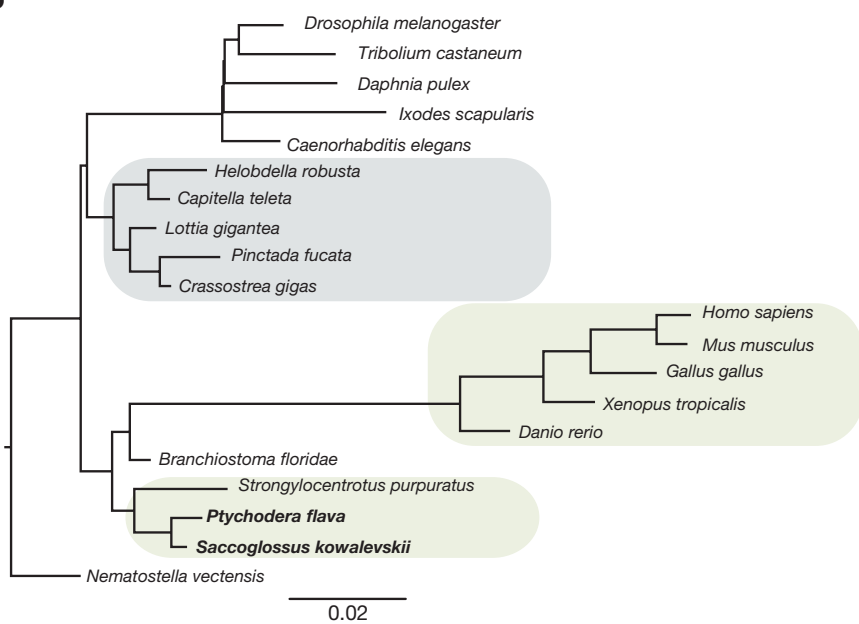

Figure 3 | High level of linkage conservation in Saccoglossus.

a, Macro-synteny dot plot between Saccoglossus and amphioxus; each dot represents two orthologous genes linked in the two species, and ordered according to their macro-syntenic linkage. Amphioxus scaffolds are organized according to the 17 ancestral linkage groups (ALGs) inferred by comparison of the amphioxus and vertebrate genomes ${ }^{27}$. Intersection areas of highest dot density are marked by numbers along the top of the plot, identifying each of the 17 putative ALGs. Axes represent orthologous gene group index along the genome. $\mathbf{b}$, Branch-length estimation for loss and gain of synteny blocks with MrBayes, see Supplementary Note 7 for details. Short branches in hemichordates (in bold) indicate a high level of micro-syntenic retention in their genomes.

pharyngeal endoderm in a band passing through the gill slit, but not localized to a thyroid-like organ ${ }^{39}$. Here we also examined the expression of $n k x 2.2$ and foxA in S. kowalevskii. We find that $n k x 2.2$, which is expressed in the ventral hindbrain in vertebrates, is expressed in pharyngeal ventral endoderm in S. kowalevskii, close to the gill slit (Fig. 4b), and that foxA is expressed throughout endoderm but repressed in the gill slit region (Fig. $4 \mathrm{~b}$ ). The co-expression of this ordered cluster of the four transcription factors during pharyngeal development strongly supports the functional importance of their genomic clustering.

The presence of this cluster in the crown-of-thorns sea star, an echinoderm that lacks gill pores, and in amniote vertebrates that lack gill slits, suggests that the cluster's ancestral role was in pharyngeal apparatus patterning as a whole, of which overt slits (perforations of a
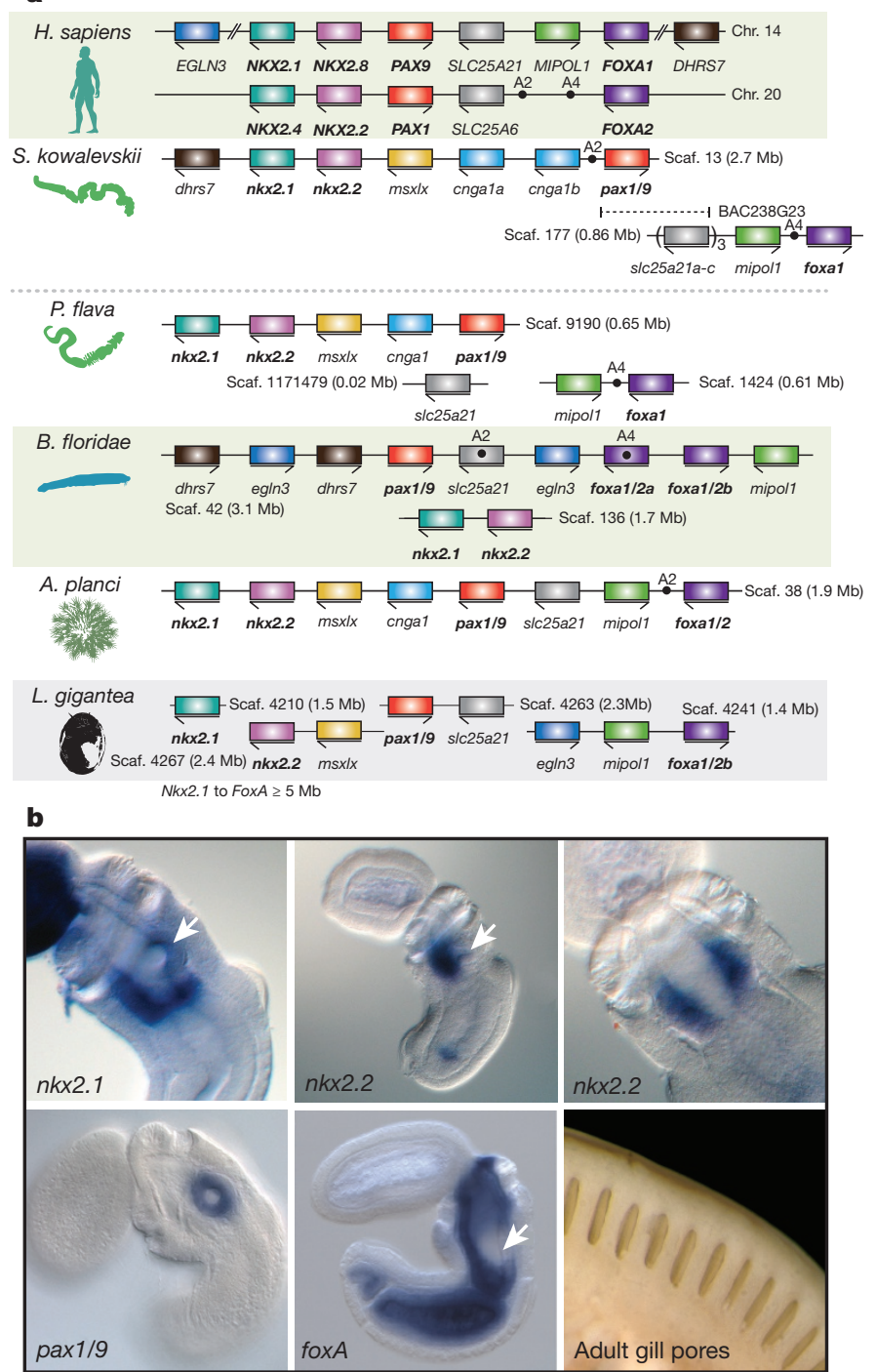

Figure 4 | Conservation of a pharyngeal gene cluster across

deuterostomes. a, Linkage and order of six genes including the four genes encoding transcription factors Nkx2.1, Nkx2.2, Pax1/9 and FoxA, and two genes encoding non-transcription factors Slc25A21 (solute transporter) and Mipol1 (mirror-image polydactyly 1 protein), which are putative 'bystander' genes containing regulatory elements of pax1/9 and foxA, respectively. The pairings of slc25A21 with pax1/9 and of mipoll with foxA occur also in protostomes, indicating bilaterian ancestry. The cluster is not present in protostomes such as Lottia (Lophotrochozoa), Drosophila melanogaster, Caenorhabditis elegans (Ecdysozoa), or in the cnidarian, Nematostella. SLC25A6 (the slc25A21 paralogue on human chromosome $20)$ is a potential pseudogene. The dots marking A2 and A4 indicate two conserved non-coding sequences first recognized in vertebrates and amphioxus ${ }^{36}$, also present in S. kowalevskii and, partially, in P. flava and A. planci. $\mathbf{b}$, The four transcription factor genes of the cluster are expressed in the pharyngeal/foregut endoderm of the Saccoglossus juvenile: $n k \times 2.1$ is expressed in a band of endoderm at the level of the forming gill pore, especially ventral and posterior to it (arrow), and in a separate ectodermal domain in the proboscis. It is also known as thyroid transcription factor 1 due to its expression in the pharyngeal thyroid rudiment in vertebrates. The $n k x 2.2$ gene is expressed in pharyngeal endoderm just ventral to the forming gill pore, shown in side view (arrow indicates gill pore) and ventral view; and pax1/9 is expressed in the gill pore rudiment itself. In $S$. kowalevskii, this is its only expression domain, whereas in vertebrates it is also expressed in axial mesoderm. The foxA gene is expressed widely in endoderm but is repressed at the site of gill pore formation (arrow). An external view of gill pores is shown; up to 100 bilateral pairs are present in adults, indicative of the large size of the pharynx. 


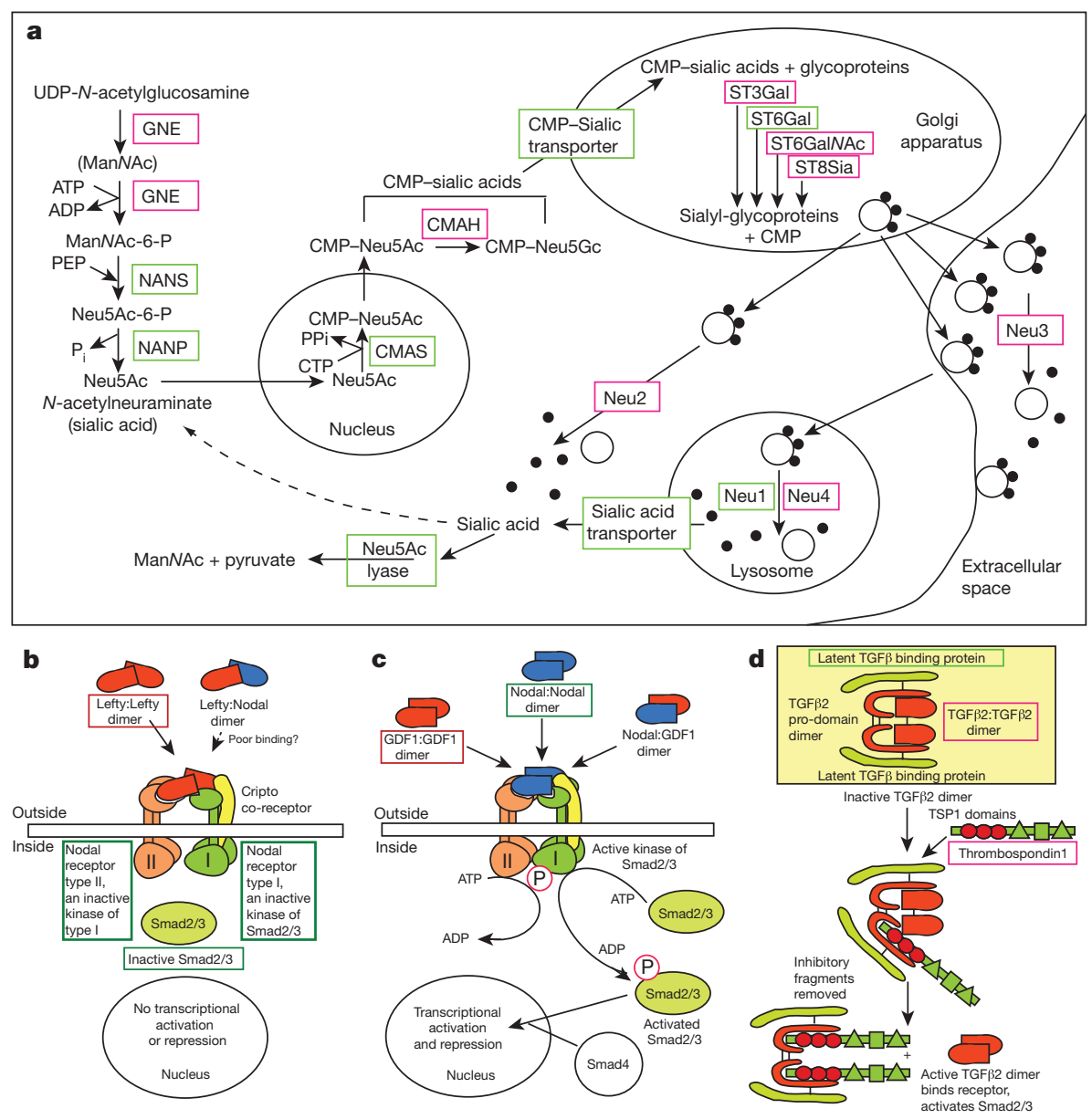

Figure 5 | Examples of deuterostome gene novelties. a, Steps of biosynthesis of sialic acid and its addition to and removal from glycoproteins. b-d, Novel genes in TGF $\beta$ signalling pathways. The encoded proteins are shown and include Lefty (b), an antagonist of Nodal signalling, which activates Smad2/3-dependent transcription when not antagonized; Univin (c), an agonist of Nodal signalling, also called Vg1, DVR1, and GDF1; and TGF $\beta 2$ (d), a ligand that activates Smad2/3dependent transcription by binding to a deuterostome-specific TGF $\beta$

apposed endoderm and ectoderm) were but one part, and the cluster is retained in these cases because of its continuing contribution to pharynx development. Genomic regions of the pharyngeal cluster have been implicated in long-range promoter-enhancer interactions, supporting the regulatory importance of this gene linkage (see Supplementary Note 9$)^{40}$. Alternatively, genome rearrangement in these lineages may be too slow to disrupt the cluster even without functional constraint. Here we propose that the clustering of the four ordered transcription factors, and their bystander genes, on the deuterostome stem served a regulatory role in the evolution of the pharyngeal apparatus, the foremost morphological innovation of deuterostomes.

\section{Deuterostome novelties}

We found $>30$ deuterostome genes with sequences that differ markedly from those of other metazoans, related to functional innovation in deuterostomes. Some plausibly arose from accelerated sequence change on the deuterostome stem from distant but identifiable bilaterian homologues, others represent new protein domain combinations in deuterostomes, while others lack identifiable sequence and domain homologues in other animals. In the latter group, we found over a dozen deuterostome genes that have readily identified relatives in marine microbes, often cyanobacteria or eukaryotic micro-algae, but are not known in other metazoans (Extended Data Table 1 and Extended Data Fig. 7; Supplementary Notes 10.4 and 10.5). Such genes receptor type II, which contains a novel ectodomain (not shown). Also shown in $\mathbf{d}$ is the novel protein thrombospondin 1 that activates TGF 32 by releasing it from an inactive complex, by way of its TSP1 domains. Red boxes around protein names indicate their deuterostome novelty. Green boxes around the names indicate genes with pan-metazoan/bilaterian ancestry and without accelerated sequence change in the deuterostome lineage.

include two of the novel deuterostome sequences associated with sialic acid metabolism (found in many microbes ${ }^{41}$, see below), enzymes that modify proteins (for example, protein arginine deiminase) and RNA (for example, FATSO methyladenosine demethylase) as well as others that provide specialized reactions of secondary metabolism (Extended Data Table 1 and Extended Data Fig. 7; Supplementary Note 10.5). Possible explanations for the unusual phylogenetic distribution of these genes include horizontal transfer on the deuterostome stem from early marine microbes (which were plausibly commensals, pathogens, or food sources of stem deuterostomes), or convergent gene loss and/or extensive sequence divergence along five or more opisthokont lineages (Supplementary Note 10.2).

Regardless of their mechanism of origination, the various deuterostome novelties and gene family expansions of sialic acid metabolism are noteworthy. Deuterostomes are unique among metazoans in their high level and diverse linkage of addition of sialic acid (also known as neuraminic acid), a nine carbon negatively charged sugar, to the terminal sugars of glycoproteins, mucins and glycolipids ${ }^{42}$. We find expanded families of enzymes for several of these reactions in hemichordates (Fig. 5a and Extended Data Table 1). Based on the presence/absence of relevant enzymes we infer that 5 of the 11 steps of the pathways of sialic acid formation, addition to termini, and removal are not found in protostomes or other metazoans, and are deuterostome novelties (Fig. 5a and Supplementary Note 10), whereas the other steps use 
enzymes similar to those of the more limited pathway of some protostomes (for example, insects such as Drosophila) ${ }^{43}$.

The importance of glycoproteins for muco-ciliary feeding and other hemichordate activities is further supported by novel and expanded families of genes encoding the polypeptide backbones of glycoproteins, those with von Willebrand type-D and/or cysteine-rich domains (PTHR11339 classifier), including mucins, present in hemichordates and amphioxus as large tandemly duplicated clusters (with varied expression patterns as shown in Extended Data Fig. 8), but not in sea urchin, which has a different mode of feeding (Supplementary Note 10). As in amphioxus, the pharynx of Saccoglossus is heavily ciliated ${ }^{44,45}$, and cells of the pharyngeal walls in hemichordates and the ventral endostyle in amphioxus secrete abundant mucins and glycoproteins ${ }^{46}$. Similarly, in the deuterostome ancestor these glycoproteins probably enhanced the muco-ciliary filter-feeding capture of food particles from the microbe-rich marine environment and protected its inner and outer tissue surfaces.

\section{Novelty in the TGF $\beta$ signalling pathway}

The signalling ligands Lefty (a Nodal antagonist) and Univin/Vg1/ $\mathrm{GDF}^{47}$ (a Nodal agonist) are deuterostome innovations that modulate Nodal signalling during the major developmental events of endomesoderm induction and axial patterning in vertebrates, axial patterning in hemichordates and echinoderms, and left-right patterning in all deuterostomes ${ }^{48}$ (see Fig. 5b-d and Extended Data Fig. 9a, b). Univin is tightly linked to the related bilaterian $b m p 2 / 4$ in the sea urchin genome $^{49}$ and also, we now report, in hemichordates and amphioxus, supporting its origin by tandem duplication and divergence from an ancestral bmp2/4-type gene, as suggested previously ${ }^{49}$.

TGF $\beta 2$ signalling (TGF $\beta 1,2$ and 3 in vertebrates) is a deuterostome innovation that controls cell growth, proliferation, differentiation and apoptosis at later developmental stages. Accompanying the novel TGF32 ligand, the type II receptor has a novel ectodomain. The extracellular matrix protein thrombospondin 1, which activates TGF $\beta 2$ in vertebrates, contains a deuterostome-unique combination of domains including three thrombospondin type 1 (TSP1) domains that bind the TGF 32 pro-domain region. While these signalling novelties have clear sequence similarity to pan-bilaterian components, they form long stem branch clades on the phylogenetic trees, indicating extensive sequence divergence on the deuterostome stem (Supplementary Note 10). Together, these innovations appear to contribute to the increased amount and complex patterning of Smad2/3-mediated signalling in deuterostomes compared with protostomes and other metazoans.

\section{Conclusion}

The two acorn worms whose genomes are described here represent the two main enteropneust lineages, separated by at least 370 million years and differing in their developmental modes. These analyses reveal (1) extensive conserved macro-synteny among deuterostomes; (2) a widely conserved deuterostome-specific cluster of six ordered genes, including four transcription factor genes that are expressed during the development of pharyngeal gill slits and the branchial apparatus, the most prominent morphological innovation of the deuterostome ancestor; and (3) numerous gene novelties shared among deuterostomes, many expanded into large families, with putative protein functions that imply physiological, metabolic and developmental specializations of the filter-feeding deuterostome ancestor. Some of these genes lack identifiable orthologues in other metazoans but do resemble microbial sequences and domain types. In addition to their contributions towards defining the deuterostome ancestor and illuminating chordate origins, the two genomes should inform hypotheses of larval evolution by providing a basis for future comparisons of direct-developing and indirect-developing acorn worms, which achieve remarkably similar adult forms by distinct embryological routes (Fig. 1).
Online Content Methods, along with any additional Extended Data display items and Source Data, are available in the online version of the paper; references unique to these sections appear only in the online paper.

Received 15 July; accepted 13 October 2015.

Published online 18 November 2015.

1. Bateson, W. The later stages in the development of Balanoglossus Kowalevskii, with a suggestion as to the affinities of the Enteropneusta. 2 parts. O. J. Microsc. Sci. 25, 81-122 (1885).

2. Bateson, W. Memoirs: the ancestry of the Chordata. Q. J. Microsc. Sci. 2, 535-572 (1886)

3. Kovalevskij, A. O. Anatomie des Balanoglossus delle Chiaje (Mémoires de I'Académie Impériale des Sciences de St. Pétersbourg: Imperatorskaja Akademija Nauk, 1866).

4. Agassiz, A. The history of Balanoglossus and tornaria. Memoirs of the American Academy of Arts and Sciences 9, 421-436 (1873).

5. Metschnikoff, V. Über die systematische Stellung von Balanoglossus. Zool. Anz 4, 139-157 (1881).

6. Halanych, K. M. The phylogenetic position of the pterobranch hemichordates based on $18 S$ rDNA sequence data. Mol. Phylogenet. Evol. 4, 72-76 (1995).

7. Cannon, J. T. et al. Phylogenomic resolution of the hemichordate and echinoderm clade. Curr. Biol. 24, 2827-2832 (2014)

8. Ogasawara, M., Wada, H., Peters, H. \& Satoh, N. Developmental expression of Pax1/9 genes in urochordate and hemichordate gills: insight into function and evolution of the pharyngeal epithelium. Development 126, 2539-2550 (1999).

9. Gillis, J. A. Fritzenwanker, J. H. \& Lowe, C. J. A stem-deuterostome origin of the vertebrate pharyngeal transcriptional network. Proc. R. Soc. Lond. B 279, 237-246 (2012).

10. Lowe, C. J., Clarke, D. N., Medeiros, D. M., Rokhsar, D. S. \& Gerhart, J. The deuterostome context of chordate origins. Nature $\mathbf{5 2 0 , 4 5 6 - 4 6 5}$ (2015).

11. Swalla, B. J. \& Smith, A. B. Deciphering deuterostome phylogeny: molecular morphological and palaeontological perspectives. Phil. Trans. R. Soc. Lond. B 363, 1557-1568 (2008)

12. Cameron, C. B., Garey, J. R. \& Swalla, B. J. Evolution of the chordate body plan: new insights from phylogenetic analyses of deuterostome phyla. Proc. Natl Acad. Sci. USA 97, 4469-4474 (2000).

13. Gerhart, J., Lowe, C. \& Kirschner, M. Hemichordates and the origin of chordates. Curr. Opin. Genet. Dev. 15, 461-467 (2005).

14. Gonzalez, P. \& Cameron, C. B. The gill slits and pre-oral ciliary organ of Protog/ossus (Hemichordata: Enteropneusta) are filter-feeding structures. Biol. J. Linn. Soc. 98, 898-906 (2009).

15. Brown, F. D., Prendergast, A. \& Swalla, B. J. Man is but a worm: chordate origins. Genesis 46, 605-613 (2008).

16. Holland, N. D., Holland, L. Z. \& Holland, P. W. Scenarios for the making of vertebrates. Nature 520, 450-455 (2015).

17. Hyman, L. H. The invertebrates: smaller coelomate groups chaetognatha, hemichordata, pogonophora, phoronida, ectoprocta, brachipoda, sipunculida, the coelomate bilateria Vol. 5. (McGraw-Hill, 1959).

18. Bourlat, S. J. et al. Deuterostome phylogeny reveals monophyletic chordates and the new phylum Xenoturbellida. Nature 444, 85-88 (2006).

19. Philippe, H. et al. Acoelomorph flatworms are deuterostomes related to Xenoturbella. Nature 470, 255-258 (2011)

20. Ruiz-Trillo, I., Riutort, M., Fourcade, H. M., Baguna, J. \& Boore, J. L. Mitochondrial genome data support the basal position of Acoelomorpha and the polyphyly of the Platyhelminthes. Mol. Phylogenet. Evol. 33, 321-332 (2004)

21. Hejnol, A. et al. Assessing the root of bilaterian animals with scalable phylogenomic methods. Proc. R. Soc. Lond. B 276, 4261-4270 (2009).

22. Edgecombe, G. D. et al. Higher-level metazoan relationships: recent progress and remaining questions. Org. Divers. Evol. 11, 151-172 (2011).

23. Srivastava, M., Mazza-Curll, K. L., van Wolfswinkel, J. C. \& Reddien, P. W. Whole-body acoel regeneration is controlled by Wnt and Bmp-Admp signaling. Curr. Biol. 24, 1107-1113 (2014).

24. Lartillot, N., Lepage, T. \& Blanquart, S. PhyloBayes 3: a Bayesian software package for phylogenetic reconstruction and molecular dating. Bioinformatics 25, 2286-2288 (2009)

25. Ulitsky, I. Shkumatava, A Jan, C. H. Sive, H. \& Bartel, D. P. Conserved function of lincRNAs in vertebrate embryonic development despite rapid sequence evolution. Cell 147, 1537-1550 (2011)

26. Royo, J. L. et al. Transphyletic conservation of developmental regulatory state in animal evolution. Proc. Natl Acad. Sci. USA 108, 14186-14191 (2011).

27. Putnam, N. H. et al. The amphioxus genome and the evolution of the chordate karyotype. Nature 453, 1064-1071 (2008)

28. Irimia, M. et al. Extensive conservation of ancient microsynteny across metazoans due to cis-regulatory constraints. Genome Res. 22, 2356-2367 (2012).

29. Sodergren, E. et al. The genome of the sea urchin Strongylocentrotus purpuratus. Science 314, 941-952 (2006).

30. Freeman, R. et al. Identical genomic organization of two hemichordate hox clusters. Curr. Biol. 22, 2053-2058 (2012) 
31. Ikuta, T. et al. Identification of an intact ParaHox cluster with temporal colinearity but altered spatial colinearity in the hemichordate Ptychodera flava. BMC Evol. Biol. 13, 129 (2013).

32. Cameron, R. A. et al. Unusual gene order and organization of the sea urchin hox cluster. J. Exp. Zoolog. B Mol. Dev. Evol. 306, 45-58 (2006).

33. Baughman, K. W. et al. Genomic organization of Hox and ParaHox clusters in the echinoderm, Acanthaster planci. Genesis 52, 952-958 (2014).

34. Santagati, F. et al. Identification of cis-regulatory elements in the mouse Pax9/Nkx2-9 genomic region: implication for evolutionary conserved synteny. Genetics 165, 235-242 (2003).

35. Lowe, C. J. et al. Dorsoventral patterning in hemichordates: insights into early chordate evolution. PLoS Biol. 4, e291 (2006).

36. Wang, W., Zhong, J., Su, B., Zhou, Y. \& Wang, Y. Q. Comparison of Pax1/9 locus reveals 500-Myr-old syntenic block and evolutionary conserved noncoding regions. Mol. Biol. Evol. 24, 784-791 (2007).

37. Santagati, F. et al. Comparative analysis of the genomic organization of Pax 9 and its conserved physical association with Nkx2-9 in the human, mouse, and pufferfish genomes. Mamm. Genome 12, 232-237 (2001)

38. Wang, S., Zhang, S., Zhao, B. \& Lun, L. Up-regulation of C/EBP by thyroid hormones: a case demonstrating the vertebrate-like thyroid hormone signaling pathway in amphioxus. Mol. Cell. Endocrinol. 313, 57-63 (2009).

39. Lowe, C. J. et al. Anteroposterior patterning in hemichordates and the origins of the chordate nervous system. Cell 113, 853-865 (2003)

40. Kokubu, C. et al. A transposon-based chromosomal engineering method to survey a large cis-regulatory landscape in mice. Nature Genet. 41, 946-952 (2009).

41. Giacopuzzi, E., Bresciani, R., Schauer, R., Monti, E. \& Borsani, G. New insights on the sialidase protein family revealed by a phylogenetic analysis in metazoa. PLOS ONE 7, e44193 (2012).

42. Harduin-Lepers, A., Mollicone, R., Delannoy, P. \& Oriol, R. The animal sialyltransferases and sialyltransferase-related genes: a phylogenetic approach. Glycobiology 15, 805-817 (2005).

43. Harduin-Lepers, A et al. Evolutionary history of the alpha2,8-sialyltransferase (ST8Sia) gene family: tandem duplications in early deuterostomes explain most of the diversity found in the vertebrate ST8Sia genes. BMC Evol. Biol. 8, 258 (2008).

44. Pardos, F. Fine structure and function of pharynx cilia in Glossobalanus minutus Kowalewsky (Enteropneusta). Acta Zoologica 69, 1-12 (1988).

45. Kaul-Strehlow, S. \& Stach, T. A detailed description of the development of the hemichordate Saccoglossus kowalevskii using SEM, TEM, Histology and 3D-reconstructions. Front. Zool. 10, 53 (2013).

46. Ruppert, E. E., Cameron, C. B. \& Frick, J. E. Endostyle-like features of the dorsal epibranchial ridge of an enteropneust and the hypothesis of dorsal-ventral axis inversion in chordates. Invertebr. Biol. 118, 202-212 (1999).

47. Range, R. \& Lepage, T. Maternal Oct1/2 is required for Nodal and Vg1/Univin expression during dorsal-ventral axis specification in the sea urchin embryo. Dev. Biol. 357, 440-449 (2011).

48. Massagué, J. TGF $\beta$ signalling in context. Nature Rev. Mol. Cell Biol. 13, 616-630 (2012).
49. Range, R. et al. Cis-regulatory analysis of nodal and maternal control of dorsal-ventral axis formation by Univin, a TGF- $\beta$ related to Vg1. Development 134, 3649-3664 (2007)

Supplementary Information is available in the online version of the paper.

Acknowledgements The Ptychodera flava genome project was supported by MEXT and OIST, Japan. This research was supported by USPHS grant HD42724 and NASA grant FDNAG2-1605 to J.G.; USPHS grant HD37277 to M.W.K.; NASA - NNX13AI68G to C.L. F.M. was funded by FP7/ERC grant [268513]. O.S. and D.S.R, and T.K. and N.S. were supported by the Molecular Genetics Unit and Marine Genomics Unit of the Okinawa Institute of Science and Technology Graduate University, respectively. Y.-H.S. and J.-K.Y. are supported by Academia Sinica and Ministry of Science and Technology, Taiwan. L.P. was supported by NIH grant R01HD073104. The Saccoglossus kowalevskii genome project was supported by a grant from the National Human Genome Research Institute, National Institutes of Health (U54 HG003273) to R.A.G.

Author Contributions J.Q., K.C.W. assembled the initial S. kowalevskii genomic assembly and performed quality assessments of the genome assemblies. Ptychodera collection, genome sequencing, and assembly: T.K., K.T., A.S., R.K., H.G., M.F., M.I.K., N.A., S.Y., A.F., T.H. Rhabdopleura collection: A.S. Saccoglossus RNA sequencing and analysis: R.M.F., M.W.K., R.C., C.L.K., S.L.L., M.H., S.R., D.M.M., K.C.W. Genome sequence production: A.C., Y.D., H.H.D., S.D., M.H., S.N.J., C.L.K., S.L.L., L.R.L., D.M., L.V.N., G.O., J.Sa., S.R., K.C.W., D.M.M., L.P., B.F., M.W.K. Saccoglossus sequence finishing: S.D., Y.D., D.M.M. Final Saccog/ossus assembly: J.J., J.Sc. Saccoglossus gene modelling and validation: T.M., J.B. J.H.F., A.M.P., M.W. Ptychodera gene modelling and analyses: T.K., R.K., K.H., E.S., F.G., K.W.B., K.T., O.S., J.G., N.S. Gene family analyses: O.S., T.K., F.M., L.P., R.M.F., C.L., J.G. Synteny: N.H.P., O.S., J.-X.Y. Repeats: O.S. Saccoglossus sequencing and assembly project management: S.R., D.M.M., K.C.W., R.A.G. Ptychodera expression analysis: Y.-C.C., Y.-H.S., J.-K.Y. Phylogenetic analyses: F.M. Additional EST collections: T.H.-K., K.T., A.S., A.T.S., J.P., P.G., C.C., C.L. HGT and novelties: J.G., O.S. Pharyngeal cluster analysis and expression: J.G., O.S., N.S., K.B., A.G. Project coordination, manuscript writing: O.S., T.K., F.M., K.T., N.S., J.G., C.L., D.S.R.

Author Information Sequencing data have been deposited in NCBI BioProject under accession number PRJNA12887 (Saccoglossus kowalevskii) and DDBJ under accession number PRJDB3182 (Ptychodera flava). Reprints and permissions information is available at www.nature.com/reprints. The authors declare no competing financial interests. Readers are welcome to comment on the online version of the paper. Correspondence and requests for materials should be addressed to O.S. (oleg.simakov@oist.jp), J.G. (jgerhart@berkeley. edu), N.S. (norisky@oist.jp) and D.S.R. (dsrokhsar@gmail.com).

(c) This work is licensed under a Creative Commons AttributionBY NA NonCommercial-ShareAlike 3.0 Unported licence. The images or other third party material in this article are included in the article's Creative Commons license, unless indicated otherwise in the credit line; if the material is not included under the Creative Commons license, users will need to obtain permission from the license holder to reproduce the material. To view a copy of this license, visit http://creativecommons.org/licenses/by-nc-sa/3.0/ 


\section{METHODS}

No statistical methods were used to predetermine sample size. The experiments were not randomized and the investigators were not blinded to allocation during experiments and outcome assessment.

Sequencing. Sperm DNA from adult males was extracted for sequencing as described in Supplementary Note 2. A single male was used for each species to minimize the impact of heterozygosity on assembly. For Saccoglossus, approximately eightfold redundant random shotgun coverage (totalling $8.1 \mathrm{~Gb}$ ) was obtained with Sanger dideoxy sequencing at the Baylor College of Medicine Genome Center, including 34,279 BAC ends and 459,052 fosmid ends. For Ptychodera, 1.3 Gb in Sanger shotgun sequences, $15.3 \mathrm{~Gb}$ in Roche 454 pyrosequence reads, and $52-\mathrm{Gb}$ paired-end sequences with Illumina MiSeq, along with mate-pairs, were generated at the Okinawa Institute of Science and Technology Graduate University. More sequencing details are available in Supplementary Note 2.

Genome assemblies. We assembled the Saccoglossus genome with Arachne ${ }^{50}$, combined with $\mathrm{BAC} /$ fosmid pair information to produce the final assembly. This Saccoglossus assembly includes 7,282 total scaffold sequences spanning a total length of $758 \mathrm{Mb}$. The relatively modest nucleotide heterozygosity $(0.5 \%)$ of S. kowalevskii, coupled with longer read lengths, enabled assembly of a single composite reference sequence. Half of the assembly is in scaffolds longer than $552 \mathrm{~kb}$ (the N50 scaffold length), and $82 \%$ of the assembled sequence is found in 1,602 scaffolds longer than $100 \mathrm{~kb}$. For Ptychodera we used the Platanus ${ }^{51}$ assembler. The resulting total scaffold length was $1,229 \mathrm{Mb}$, with half the assembly in scaffolds longer than $196 \mathrm{~kb}$ (N50 scaffold length). P. flava exhibited a notably higher heterozygosity ( $1.3 \%$ single nucleotide heterozygosity with frequent indels) than S. kowalevskii, presumably related to its pelagic dispersal and larger effective population size ${ }^{52}$. We therefore initially produced stringent separate assemblies of the two divergent haplotypes, and found that many scaffolds had a closely related second scaffold with 94\% BLASTN identity (over longer stretches, including indels). To avoid reporting both haplotypes at these loci, scaffolds with less than $6 \%$ divergence over at least $75 \%$ of their length were merged into a single haploid reference for comparative analysis. To further classify regions with 'double' depth and single haplotype regions we implemented a Hidden Markov Model classifier. We find that at least $63 \%$ of the initial Platanus assembly constitutes merged haplotypes. The inferred SNP rate for those regions is $1.3 \%$, while for the remaining haplotype regions it is below $0.1 \%$. Further details of assemblies are described in Supplementary Note 2.

Gene predictions. Transcriptome data for both species were used, along with homology-guided and $a b$ initio methods, to predict protein-coding genes (Supplementary Note 3). For Saccoglossus, 8.6 million RNAseq reads were generated from 7 adult tissues and 15 developmental stages using Roche 454 sequencing, along with previously deposited ESTs in GenBank. For Ptychodera, extensive EST data from egg, blastulae, gastrulae, larvae, juveniles, adult proboscis, stomochord, and gills defining 34,159 cDNA clones ${ }^{53}$, and 879,000 Roche/454 RNAseq reads from a mixed library of developmental stages ${ }^{54}$ were used. The Saccoglossus genome was annotated using JGI gene prediction pipeline ${ }^{55}$, while Augustus ${ }^{56}$ was used to produce gene models for Ptychodera. We find a total of 34,239 gene predictions for Saccoglossus (68\% with transcript evidence) and 34,687 for Ptychodera ( $43 \%$ with transcript evidence), although these are overestimates of the true gene number due to fragmented gene predictions, mis-annotated repetitive sequences, and spurious predictions. As described in the main text, 18-19,000 gene models in each species have known annotations and/or orthologues in other species.

Gene family analysis. Gene family clustering was done using a progressive (leaf to root) BLASTP-based clustering algorithm, where at a given phylogenetic node the gene families are constructed taking into account protein similarities among ingroups and outgroups ${ }^{57}$. For the inference of deuterostome gene families we use the bilaterian node of the clustering. To call gene families present in the deuterostome ancestor, we required (1) at least two ambulacrarian orthologues out of the three available ambulacrarian genomes and at least two chordate orthologues, or (2) at least two deuterostomes (chordates and/or ambulacrarians) and two outgroups in the bilaterian level clusters.

Transposable elements. Repetitive sequences were identified using RepeatScout ${ }^{58}$, followed by manual curation and annotation using both a Repbase release (version $20140131)^{59}$ and BLASTX-based search against a custom collection of transposons, using a previously described repeat identification and annotation pipeline ${ }^{57}$ (Supplementary Note 5). The assemblies were then masked with RepeatMasker version open-4.0.5 $5^{60}$. The repetitive complements of the two hemichordate genomes are summarized in Supplementary Table 5.1.

Phylogenetic analysis. Phylogenetic analyses were done using metazoan-level gene family clusters based on whole-genome sequences (Supplementary Note 4), selecting a single orthologue per genome with the best cumulative BLASTP to other species, and best reciprocal BLASTP hits to species with transcriptome-only information (Supplementary Note 6). Single gene alignments were built using Muscle $^{61}$ and filtered using Trimal ${ }^{62}$ for each orthologue, and were concatenated, yielding a supermatrix of 506,428 positions with $34.9 \%$ missing data. This supermatrix was analysed with ExaML assuming a site-homogenous $\mathrm{LG}+\Gamma_{4}$ model partitioned for each gene ${ }^{63}$. A slow-fast analysis was conducted to stratify marker genes based on the length of the branch leading to acoels in individual trees. A subset of the slowest $10 \%$ of genes was analysed with the site-heterogenous $\mathrm{CAT}+\mathrm{GTR}+\Gamma_{4}$ model using Phylobayes ${ }^{24}$. Molecular dating was carried out using Phylobayes ${ }^{24}$ using the log-normal relaxed clock model and the calibrations described in Supplementary Table 6.2.

Synteny analysis. Macro- and micro-syntenic linkages were calculated as described in Supplementary Note 7. For Fig. 3a, we merged the amphioxus scaffolds into 17 pre-defined scaffold groups as suggested in ref. 27. These 17 merged scaffold groups represent the 17 ancestral linkage groups (ALGs) shared in chordates. Then we calculated the orthologous gene groups shared by each amphioxus ALG-Saccoglossus scaffold pair and generated the dot plot as described in Supplementary Note 7. For micro-synteny we required at least three genes (separated by a maximum of ten genes) to be present in pairwise comparisons. Under random reshuffling of the genome, this yields $10 \%$ false positives in pairwise genome comparisons, that is, we observe approximately one-tenth as many micro-syntenic blocks between the two genomes when gene orders are shuffled. This false-positive rate, however, falls to $1 \%$ when considering more than two species. For our inference of deuterostome ancestral and novel synteny we therefore focus on blocks present in at least three species (and both ingroup representatives, that is, ambulacrarians and chordates). This yields 698 blocks that can be traced back to the deuterostome ancestor, including 71 blocks found exclusively in deuterostome species (shared among ambulacrarians and chordates), including the pharyngeal cluster discussed in Fig. 4.

Whole-genome alignment. Whole-genome alignments were conducted with MEGABLAST ${ }^{64}$ using parameters previously reported ${ }^{65}$. We assessed the distribution of the resulting 12,722 aligned loci across known gene annotations in ENSEMBL $^{66}$, previously identified conserved pan-vertebrate element ${ }^{65}$, as well as known enhancers in human according to LBL database ${ }^{67}$.

Gene novelties. Deuterostome gene novelties were assessed initially through bilaterian gene clusters (Supplementary Note 10) by requiring at least two species on both ambulacrarian and chordate side to be present. The novelties were further automatically subdivided into four categories: G1 (gain type I), with no BLASTP hit outside of deuterostomes; G2 (gain type II), with a novel PFAM domain present only in deuterostomes; G3 (gain type III) having a novel PFAM combination unique to deuterostomes; and G4 (gain type IV), those that do not fall under any of the G1-3 categories and define novelties due to acceleration in the substitution rate on the deuterostome stem. To confirm the novel nature, especially for G4 novelties, we have constructed phylogenies for the members and non-deuterostome BLASTP hits (up to an $e$-value of $1 \times 10^{-20}$ ) using MAFFT-alignment-based FastTree calculations. The trees were assessed for the accelerated rate of evolution at the deuterostome stem (Supplementary Fig. 9.1.1). The final result is provided in the Supplementary Information.

Curation of candidates for horizontal gene transfer on the deuterostome stem. We examined in detail gene families found broadly in deuterostomes whose encoded peptides were readily alignable to microbial sequences but had no detectable similarity in non-deuterostome animals. Criteria for evaluation included: (1) the hemichordate gene matches microbial genes at least ten orders of magnitude in the $e$-value better than it matches sequences of non-deuterostome metazoan (most of the putative HGTs we describe have no non-deuterostome metazoan hit at all); (2) it has a defined genomic locus among bona fide metazoan genes; (3) it shares an exon-intron structure with genes of chordates and other ambulacraria; and (4) when a low bitscore match is found to a non-deuterostome metazoan sequence, that sequence is identified as containing different domains (domain structure according to $\mathrm{CDD}^{68}$ ) and/or different exon-intron structure, implying dubious relatedness. When phylogenetic trees are constructed for these HGTcandidate proteins, the trees contain numerous branches for microbial sequences and none for non-deuterostome metazoan sequences, or only very long branches for dubiously relatives, and hence the trees differ greatly from the metazoan species tree, except within the deuterostome clade.

Code availability. Original data and code can be accessed at https://groups.oist. jp/molgenu.

50. Jaffe, D. B. et al. Whole-genome sequence assembly for mammalian genomes: Arachne 2. Genome Res. 13, 91-96 (2003).

51. Kajitani, R. et al. Efficient de novo assembly of highly heterozygous genomes from whole-genome shotgun short reads. Genome Res. 24, 1384-1395 (2014).

52. Romiguier, J. et al. Comparative population genomics in animals uncovers the determinants of genetic diversity. Nature 515, 261-263 (2014). 
53. Tagawa, K. et al. A cDNA resource for gene expression studies of a hemichordate, Ptychodera flava. Zoolog. Sci. 31, 414-420 (2014).

54. Chen, S. H. et al. Sequencing and analysis of the transcriptome of the acorn worm Ptychodera flava, an indirect developing hemichordate. Mar. Genomics 15, 35-43 (2014).

55. Salamov, A. A. \& Solovyev, V. V. Ab initio gene finding in Drosophila genomic DNA. Genome Res. 10, 516-522 (2000)

56. Stanke, M. \& Waack, S. Gene prediction with a hidden Markov model and a new intron submodel. Bioinformatics 19 (Suppl. 2), ii215-ii225 (2003).

57. Simakov, O. et al. Insights into bilaterian evolution from three spiralian genomes. Nature (2013).

58. Price, A. L., Jones, N. C. \& Pevzner, P. A. De novo identification of repeat families in large genomes. Bioinformatics 21 (Suppl. 1), i351-i358 (2005).

59. Jurka, J. et al. Repbase Update, a database of eukaryotic repetitive elements. Cytogenet. Genome Res. 110, 462-467 (2005).

60. Smit, A., Hubley, R. \& Green, P. RepeatMasker http://www.repeatmasker.org. (2007).

61. Edgar, R. C. MUSCLE: a multiple sequence alignment method with reduced time and space complexity. BMC Bioinformatics 5, 113 (2004).
62. Capella-Gutiérrez, S., Silla-Martinez, J. M. \& Gabaldon, T. trimAl: a tool for automated alignment trimming in large-scale phylogenetic analyses. Bioinformatics 25, 1972-1973 (2009).

63. Aberer, A. \& Stamatakis, A. ExaML: Exascale maximum likelihood: program and documentation. See http://sco.h-its.org/exelixis/web/software/examl/index. html (2013).

64. Altschul, S. F. et al. Gapped BLAST and PSI-BLAST: a new generation of protein database search programs. Nucleic Acids Res. 25, 3389-3402 (1997).

65. Lee, A. P., Kerk, S. Y., Tan, Y. Y., Brenner, S. \& Venkatesh, B. Ancient vertebrate conserved noncoding elements have been evolving rapidly in teleost fishes. Mol. Biol. Evol. 28, 1205-1215 (2011)

66. Cunningham, F. et al. Ensembl 2015. Nucleic Acids Res. 43, D662-D669 (2015)

67. Visel, A., Minovitsky, S., Dubchak, I. \& Pennacchio, L. A. VISTA Enhancer Browser-a database of tissue-specific human enhancers. Nucleic Acids Res. 35, D88-D92 (2007).

68. Marchler-Bauer, A. et al. CDD: NCBl's conserved domain database. Nucleic Acids Res. 43, D222-D226 (2015).

69. Marinić, M., Aktas, T., Ruf, S. \& Spitz, F. An integrated holo-enhancer unit defines tissue and gene specificity of the Fgf8 regulatory landscape. Dev. Cell 24, 530-542 (2013) 


\section{RESEARCH ARTICLE}

a

\section{Saccoglossus}

Scaffold total

Contig total

Scaffold sequence total, Mb

Scaffold N50, kb

Gene models

SNP rate, \%
7,282

20,913

758

552

34,239

0.5

\section{Ptychodera}

218,255

322,077

1,229

196

34,687

$1.3(2 x), 0.06(1 x)$ b

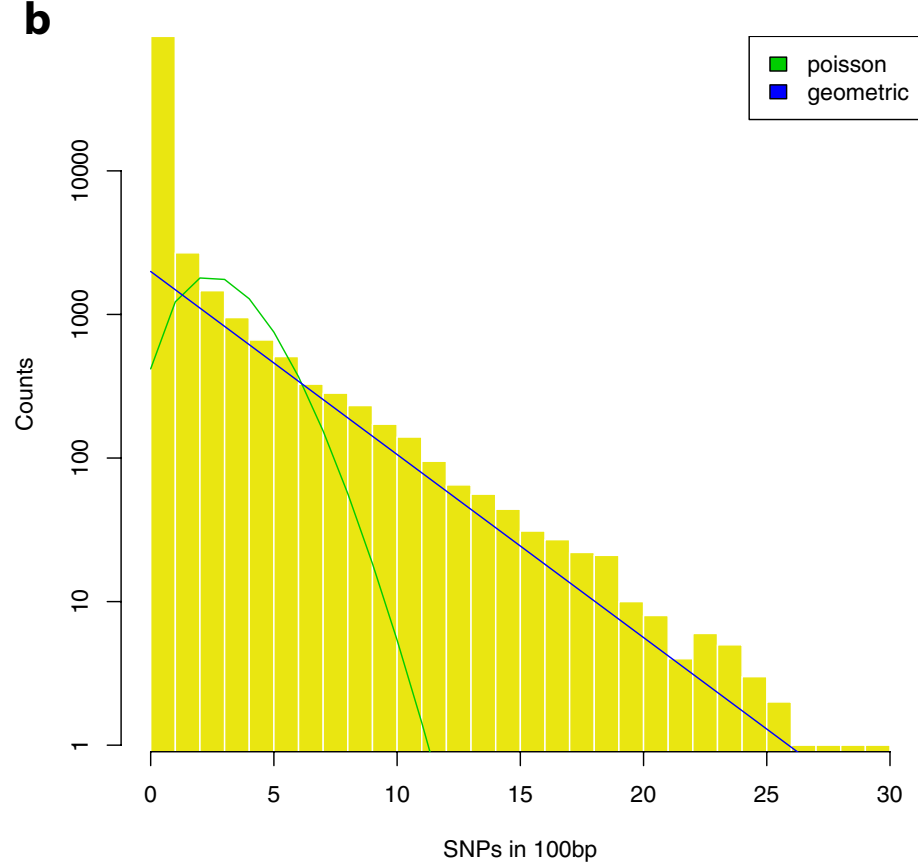

C

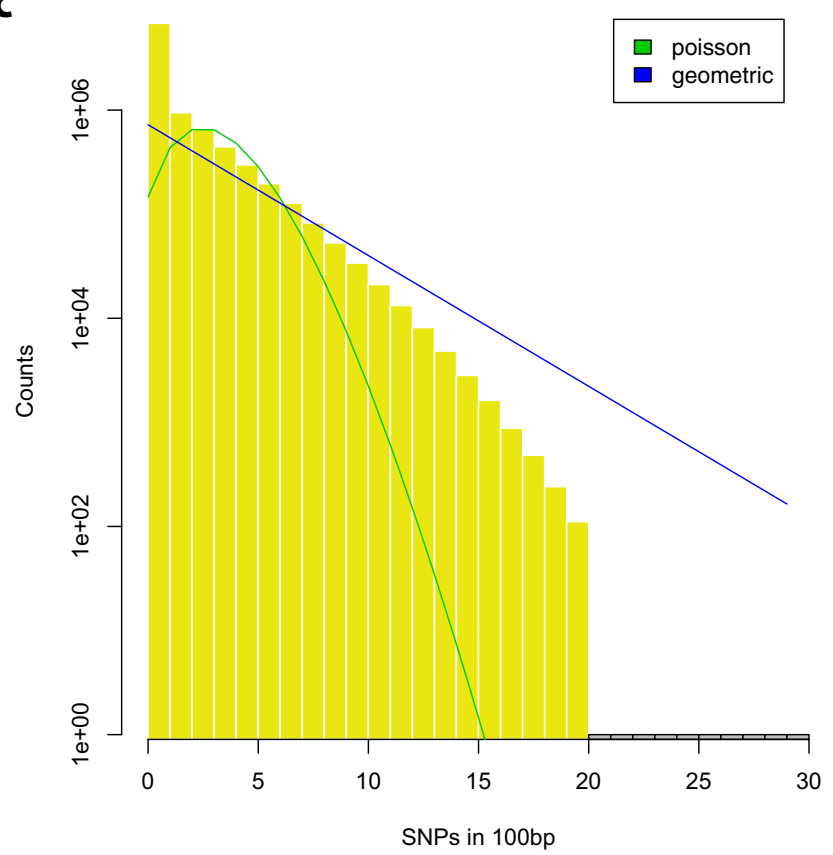

is present) and a Poisson distribution (expected with low recombination rate). The distribution for Saccoglossus is fitted to windows with one or more SNPs only, as there is an excess of zero SNP windows (approximately $84 \%$ of total 94,324 selected windows). For methods refer to Supplementary Note 2 .
Extended Data Figure 1 | Summary of genome assemblies and heterozygosity distributions for Saccoglossus and Ptychodera. a, Genome statistics summary. b, c, The single nucleotide polymorphism distribution across 100-bp windows for Saccoglossus (b) and the corresponding distribution for Ptychodera (c). The distributions in $\mathbf{b}$ and $\mathbf{c}$ are fitted with a geometric (expected when high recombination rate 


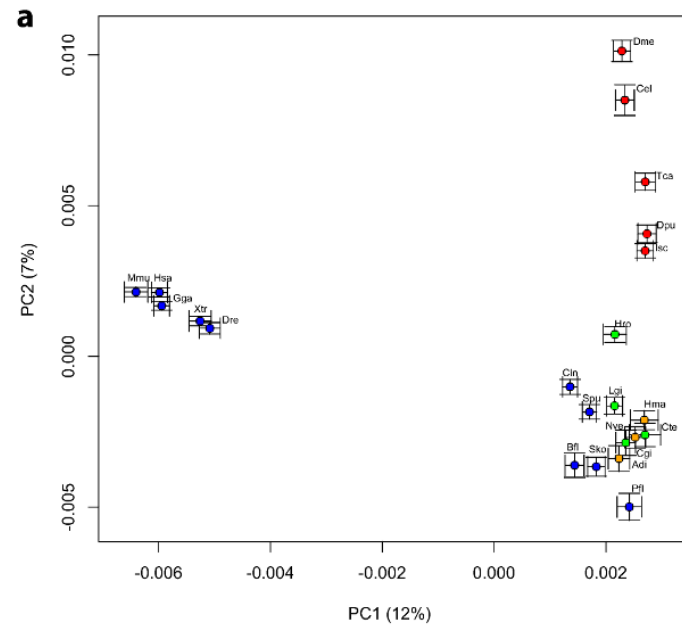

Extended Data Figure $2 \mid$ Ambulacrarians approximate the ancestral metazoan gene repertoire. a, Principal component analysis of Panther gene family sizes. Variances of the first two components are plotted in parentheses. Blue indicates deuterostomes; green indicates lophotrochozoans; red, ecdysozoans; yellow/orange, non-bilaterian metazoans. Note the clustering of the ambulacrarians Sko, Pfl and $\mathrm{Spu}$ with the non-vertebrate deuterostomes $\mathrm{Bfl}$ and $\mathrm{Cin}$ in the lower b

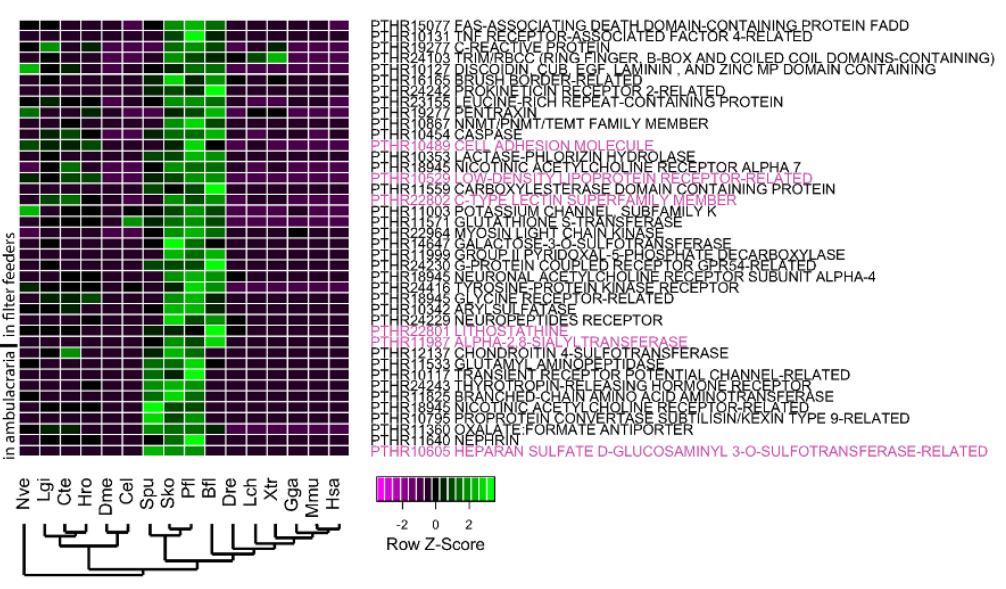

right corner, also with the lophotrochozoans Cgi, Lgi, Hro, Cte and the non-bilaterians Hma, Nve and Adi. b. Heat map of gene family counts showing significant (Fisher's exact test $P$ value $<0.01$ after Bonferroni multiple testing correction) expansion in ambulacrarians as well as in Saccoglossus/Ptychodera/amphioxus. The cases discussed in the main text are highlighted in red. See Supplementary Note 4 for details. Species abbreviations are defined in Supplementary Note 4.1. 


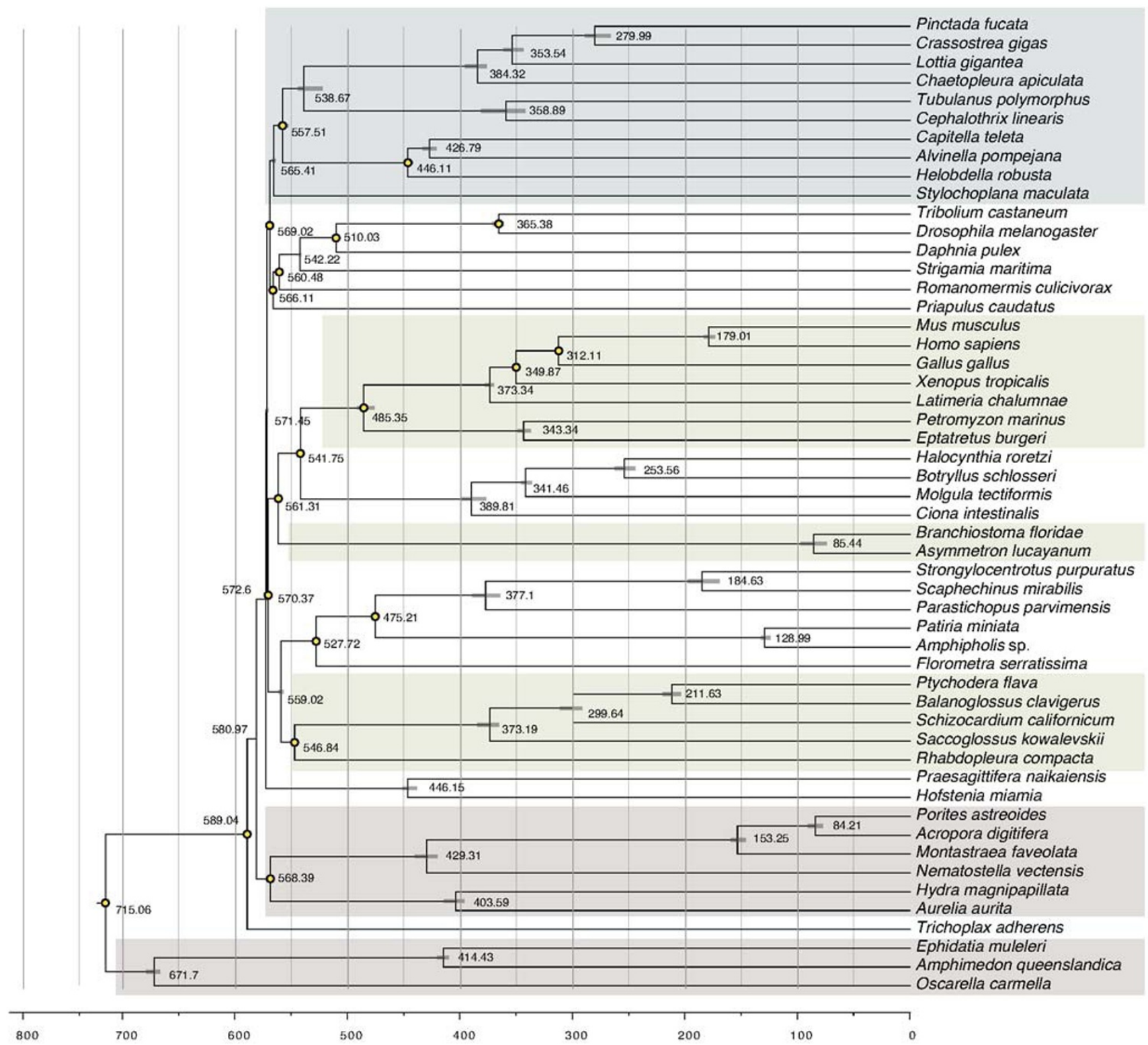

Extended Data Figure $3 \mid$ Molecular dating of deuterostome and metazoan radiations using PhyloBayes assuming a log-normal relaxed clock model. Yellow circles on particular nodes indicate the calibration dates applied from the fossil record, as indicated in Supplementary Note 6.2. Bars are $95 \%$ credibility intervals derived from posterior distributions.
Note the estimated times of divergence of chordates and ambulacraria (the deuterostome ancestor) at 570 million years ago (Ma; midEdiacaran), hemichordates and echinoderms at $559 \mathrm{Ma}$, enteropneusts and pterobranchs at $547 \mathrm{Ma}$, and Harrimaniid and Ptychoderid enteropneusts at $373 \mathrm{Ma}$. 

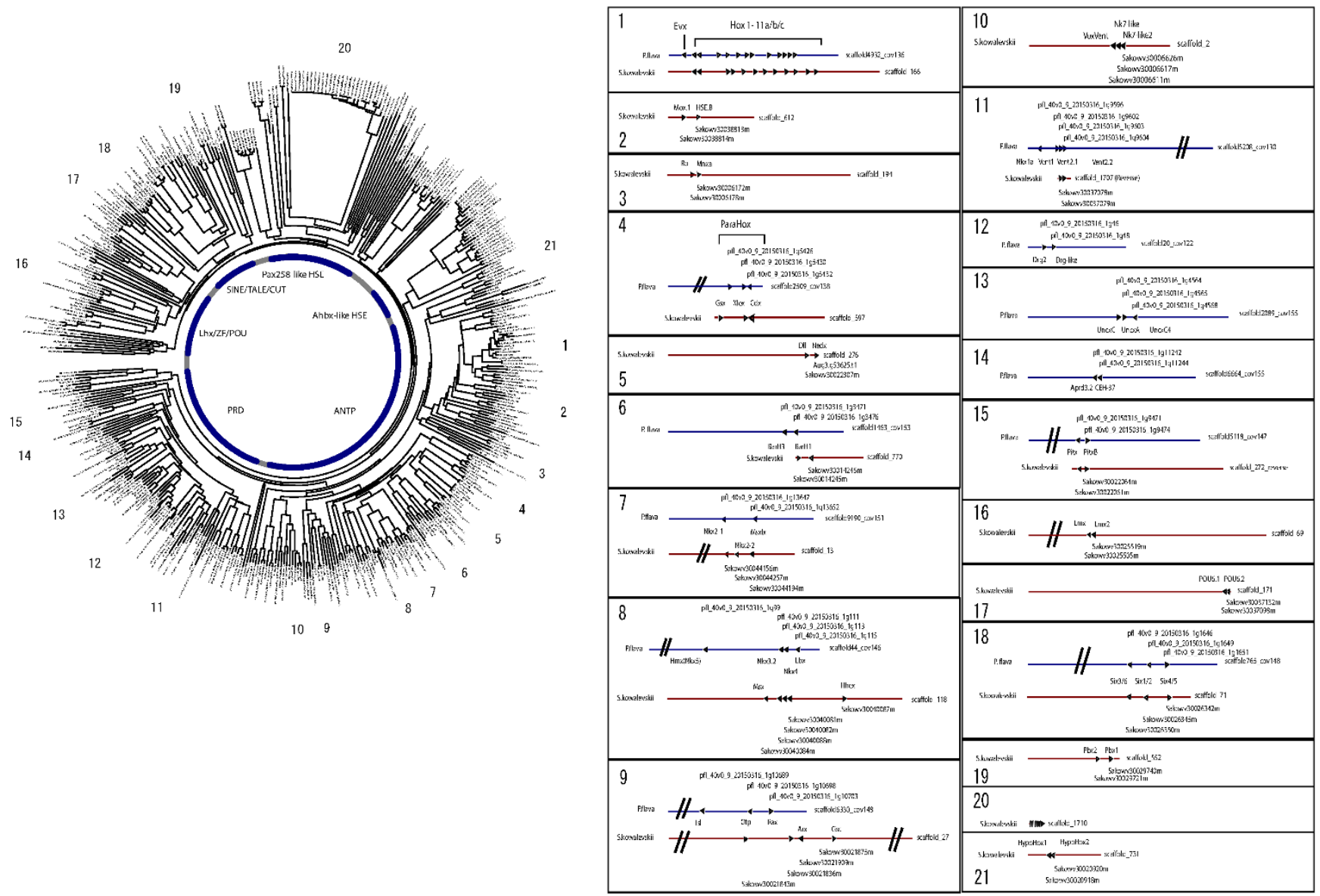

Extended Data Figure 4 | Homeobox gene complement of the two hemichordates in comparison to that of amphioxus. The numbers of homeobox-containing gene models are 170 in Saccoglossus and 139 in Ptychodera. These homeobox domains were aligned with 128 homeobox genes of Branchiostoma floridae using ClustalW2, then gaps and unaligned regions were manually removed. Since some genes have more than one homeobox domain, we kept all domains or chose the longest one according to the state of domain conservation. In total, 448 homeobox sequences were aligned. See Supplementary Information for details. The clusters of homeobox genes on scaffolds in Saccoglossus and Ptychodera were identified and drawn at positions around the tree. Conserved clusters between the two species were aligned. In addition to the well-known Hox and ParaHox cluster, 17 clusters were found in at least one of the hemichordates or some in both. Sixteen genes of the Nkx class are distributed over four clusters: (i) nkx1a-vent1-vent2.1-vent2.2; (ii) $n k x 2.1-n k x 2.2-m s x l x$; (iii) $n k x 5-m s x-n k x 3.2-n k x 4-l b x-h e x$; and (iv) voxvent-nk7like-nk7like2. The second cluster (ii) of these is part of the pharyngeal cluster (Fig. 4). Another five-gene cluster consists of one Lim class homeobox gene and four PRD class homeobox genes; isl-otp-raxarx-gsc. A cluster of six3/6-six1/2-six4/5 was found in both species, and a cluster of three $u n x$ genes was found only in P. flava. Ten more clusters were found containing two homeobox genes each. Notably, we found species-specific homeobox clusters in both species. Three remarkable clusters were found in S. kowalevskii in which 10,12 and 5 homeobox-containing genes are tandem duplicated in scaffold_1710,_52 and_4796, respectively. We also found such clusters in P.flava in which $7,4,8$ and 10 genes are aligned on scaffold 19451, scaffold 1398, scaffold 12422 and scaffold 154657, respectively. All homeobox genes identified in the genomes of the two hemichordates and amphioxus are listed in the Supplementary Table for Extended Data Fig. 4. This list includes some genes not containing a homeobox (for example, pax1/9) in cases where other family members do (for example, pax2). 


\section{RESEARCH ARTICLE}

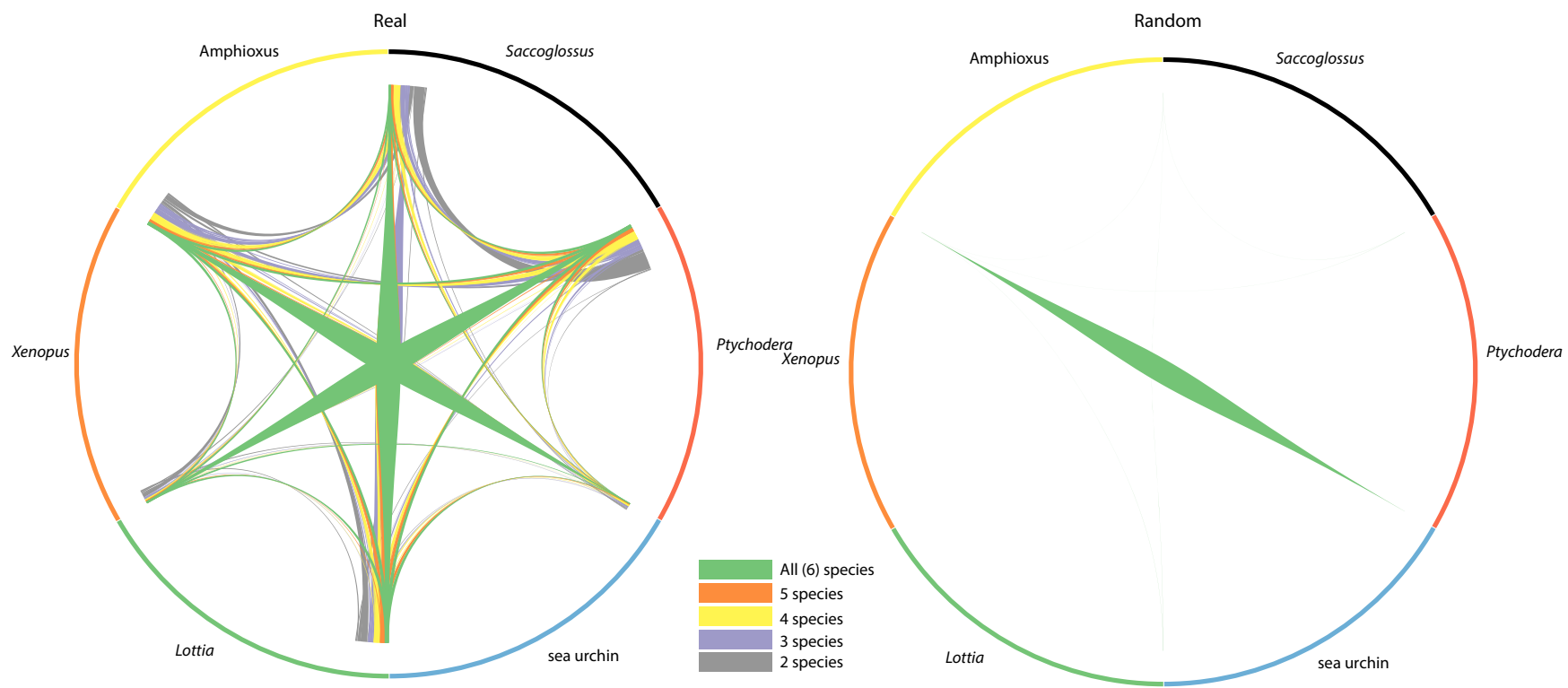

Extended Data Figure $5 \mid$ High retention rate of micro-synteny in Saccoglossus. Circos plot showing micro-syntenic conservation in blocks of genes $\left(n_{\max }=10\right.$ and $\left.n_{\min }=2\right)$ for six metazoan species for observed (left) and simulated (right) linkages. The width of connecting segments is proportional to the number of genes participating in the syntenic linkages (normalized by the total gene count). In this representation scaffolds are placed end-to-end, and adjacent scaffolds need not be from the same chromosome. While simulated data yields some blocks shared between pairs of species, few or no synteny blocks can be recovered among three or more species (Methods). Saccoglossus shows one of the highest retentions among the selected species (and the highest among the sequenced ambulacrarians). Xenopus (and vertebrates in general) have lost some micro-synteny due to whole-genome duplications and differential loss of paralogues. The matching between the hemichordate S. kowalevskii and the chordate amphioxus is highest, consistent with the fact that neither genome has undergone extensive gene loss (as have tunicates) or pseudotetraploidization with extensive loss of paralogues (as have vertebrates). 
a

Hsa chr_13 27250kb

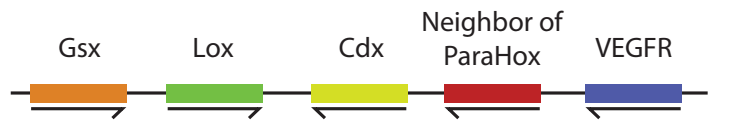

$27500 \mathrm{~kb}$

Sko scaffold_597 0kb

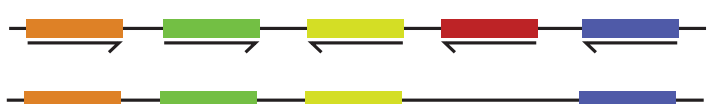

$250 \mathrm{~kb}$

Pfl scaffold_2509 747kb

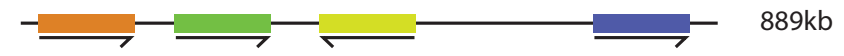

b

Sko scaffold_96

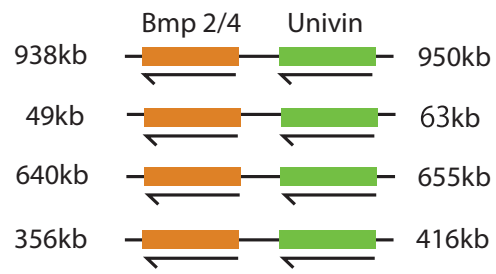

Pfl scaffold_11057

Bfl scaffold_347

Spu NW001346260

$356 \mathrm{~kb}$

$416 \mathrm{~kb}$

\section{C}

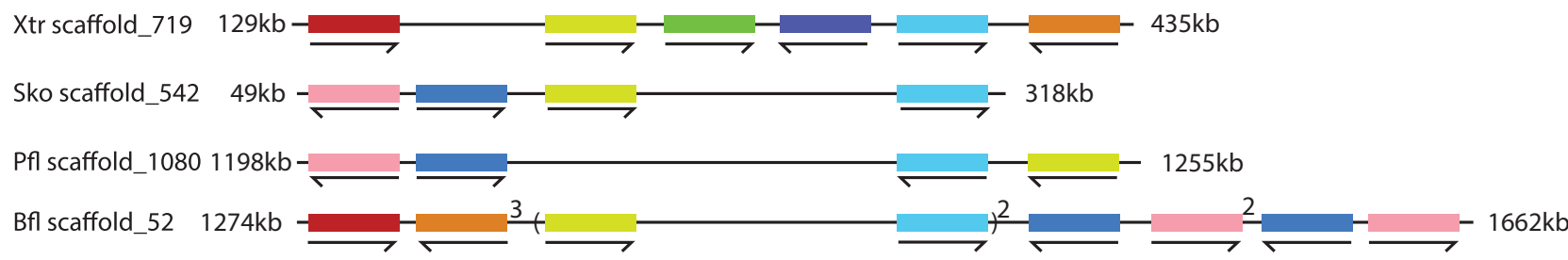

PTHR1 1848:SF7 Lefty

PTHR10050:SF11 Protein O-Mannosyl-Transferase

PTHR22973:SF6 Golgi residient protein

PTHR21661:SF2 Epoxide Hydrolase 1

PTHR12786:SF1 Uncharacterized Splicing Factor

PTHR11260:SF59 Glutathione S-Transferase

PTHR13018:SF9 Uncharacterized Transmembrane Protein

PTHR10662:SF18 Nuclear RNA Export Factor 1

\section{d}

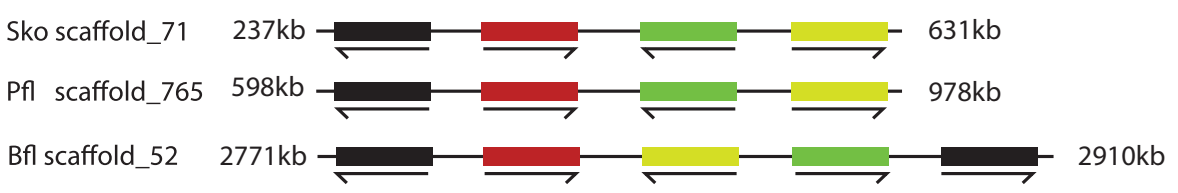

PTHR19282:SF69 Tetraspanin-like PTHR10390:SF13 Homeobox Protein Six1 PTHR10390:SF16 Homeobox Protein Six4 PTHR10342:SF28 Arylsulfatase
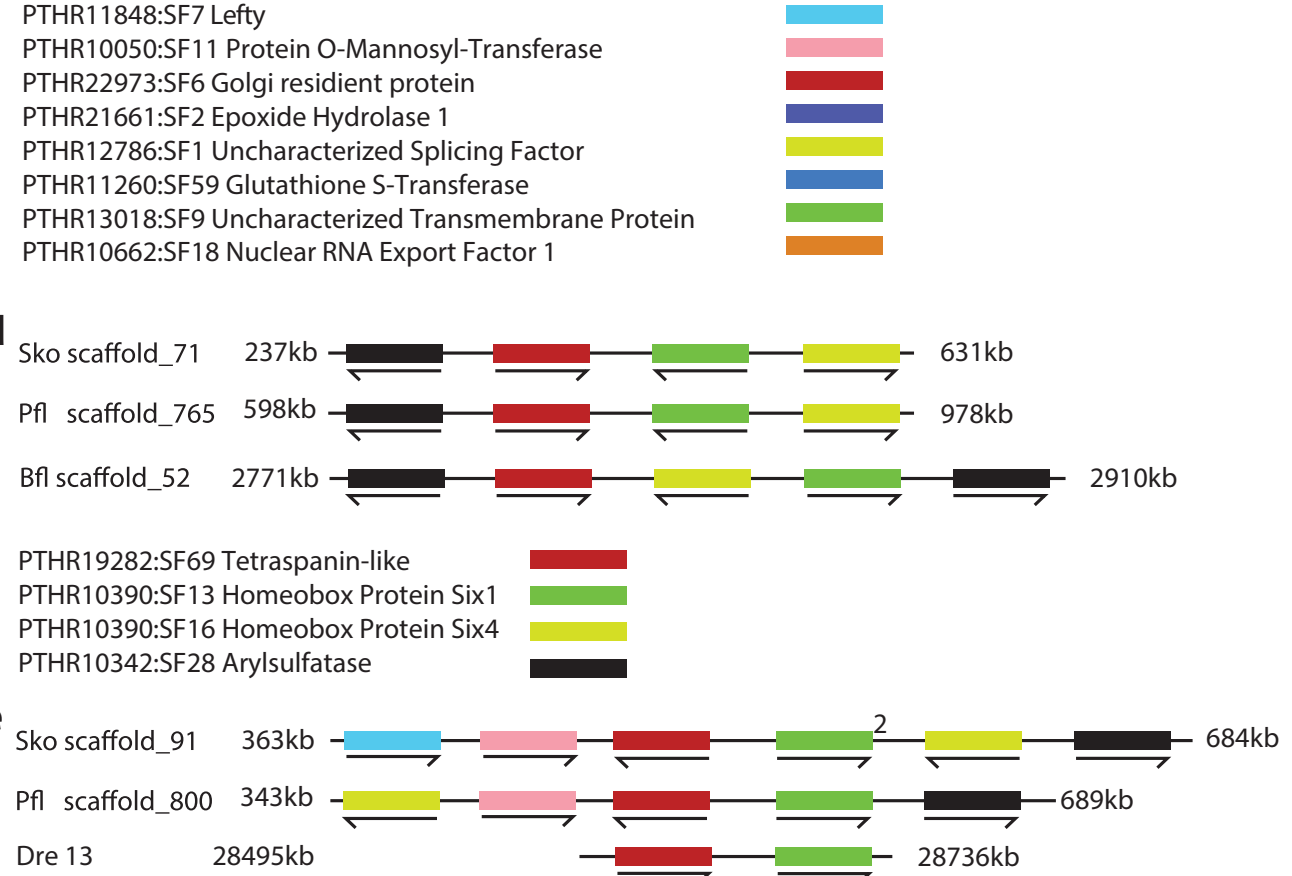

Hsa 10

$101800 \mathrm{~kb}$

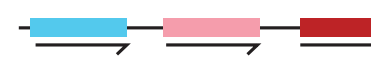

PTHR1 1486:SF2 Fibroblast Growth Factor 8

PTHR14381:SF0 F-box/WD repeat domain containing protein PTHR19316:SF1 Nucleotide exchange factor SIL1

PTHR24082:SF13 Peroxisome Proliferator-activated Receptor Alpha

PTHR23055:SF55 KCNIP K channel-interacting protein 1

PTHR22747 Nucleoplasmin

Extended Data Figure 6 Deuterostome specific micro-syntenic

linkages. a, b, Very tight linkages with no intervening genes. a, ParaHox cluster shown in S. kowalevskii, P. flava, and human. b, bmp2/4 and univin cluster in the hemichordates $S$. kowalevskii and P. flava, the sea urchin S. purpuratus, and the cephalochordate B. floridae.

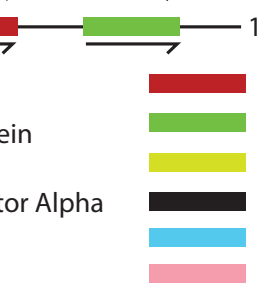

c-e, Loose micro-syntenic linkages with a maximum of five intervening genes: lefty (c), six1-six4 (d), and $f g f 8-f b x w(\mathbf{e})^{69}$ clusters. For $\mathbf{c}$ to $\mathbf{e}$ all species with micro-synteny are shown. Numbers above the genes indicate the copy number in the locus. 
a

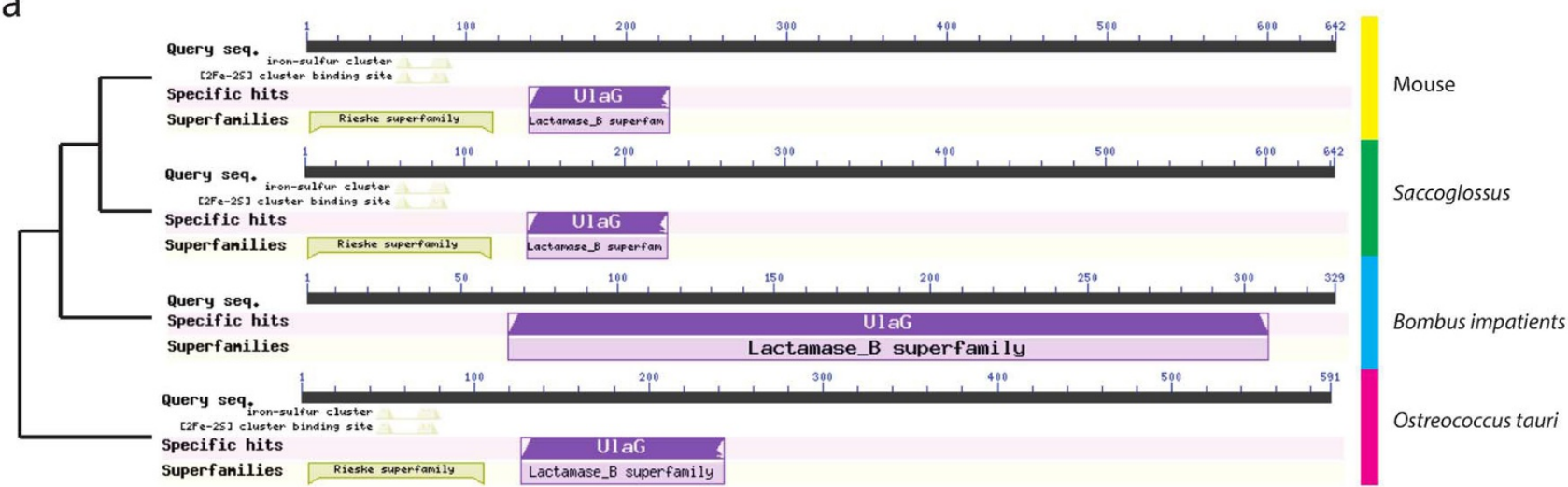

b

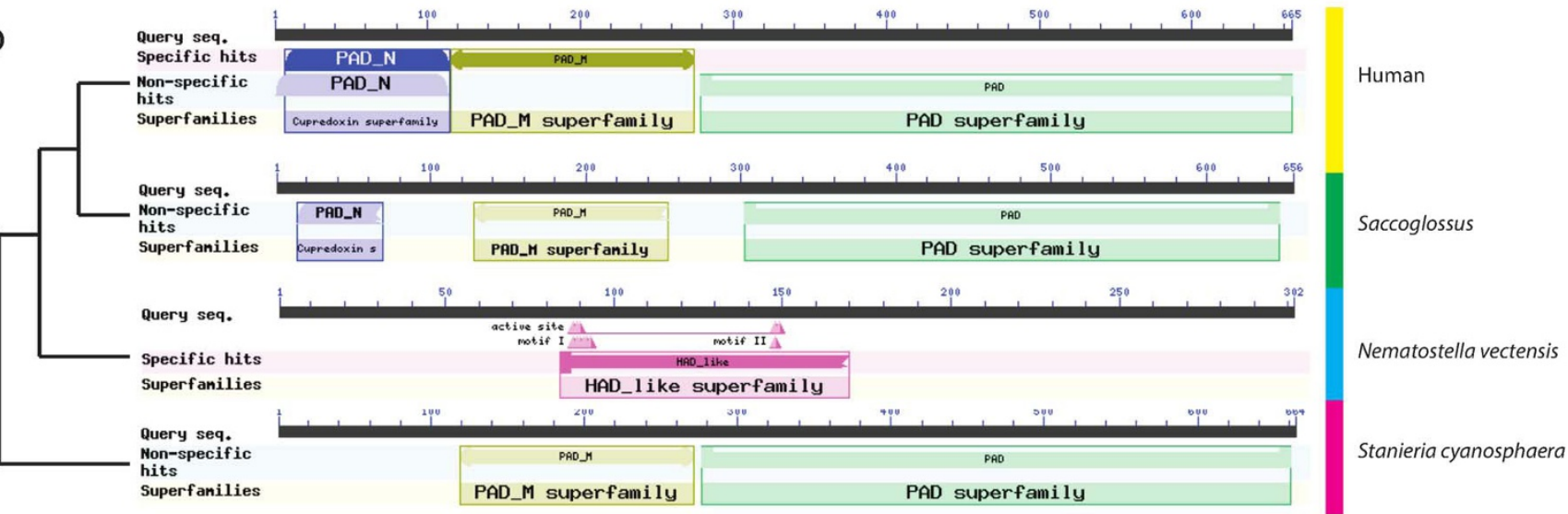

C
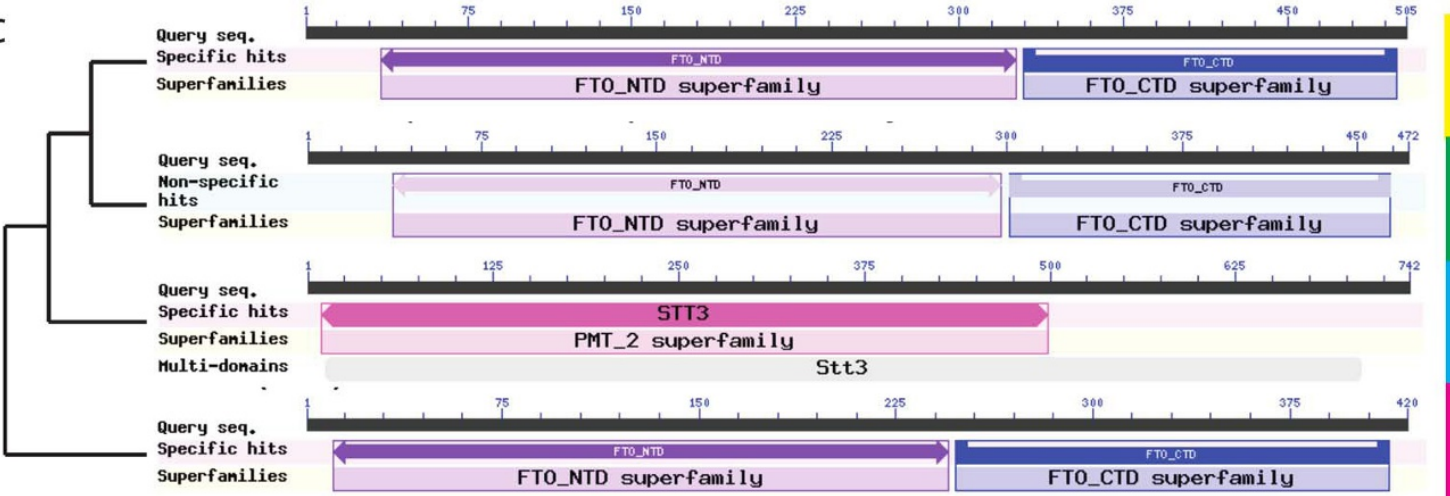

Human

Saccoglossus

Lottia gigantea

Ostreococcus tauri

Extended Data Figure 7 | Three examples showing the domain structures of some proteins encoded by genes found in deuterostomes and marine microbes but not non-deuterostome animals. Best BLASTP hits of the Saccoglossus sequence in human/mouse, as well as in non-deuterostome metazoans and in non-metazoans (such as the cyanobacterium Staniera cyanosphaera, or the eukaryotic micro-alga Ostreococcus tauri) are shown. a, Cytidine monophosphate- $N$-acetylneuraminic acid hydroxylase (CMAH), an enzyme of sialic acid modification; $\mathbf{b}$, peptidyl arginyl deiminase (PAD), an enzyme of post-translational modification of proteins; c, FATSO-like, also called $\alpha$-ketoglutarate-dependent dioxygenase FTO, an enzyme that de-methylates $N^{6}$-methyladenosine in nuclear RNA. Other analyses of these and other genes with the unusual phylogenetic distributions can be found in Supplementary Note 10 . 
a
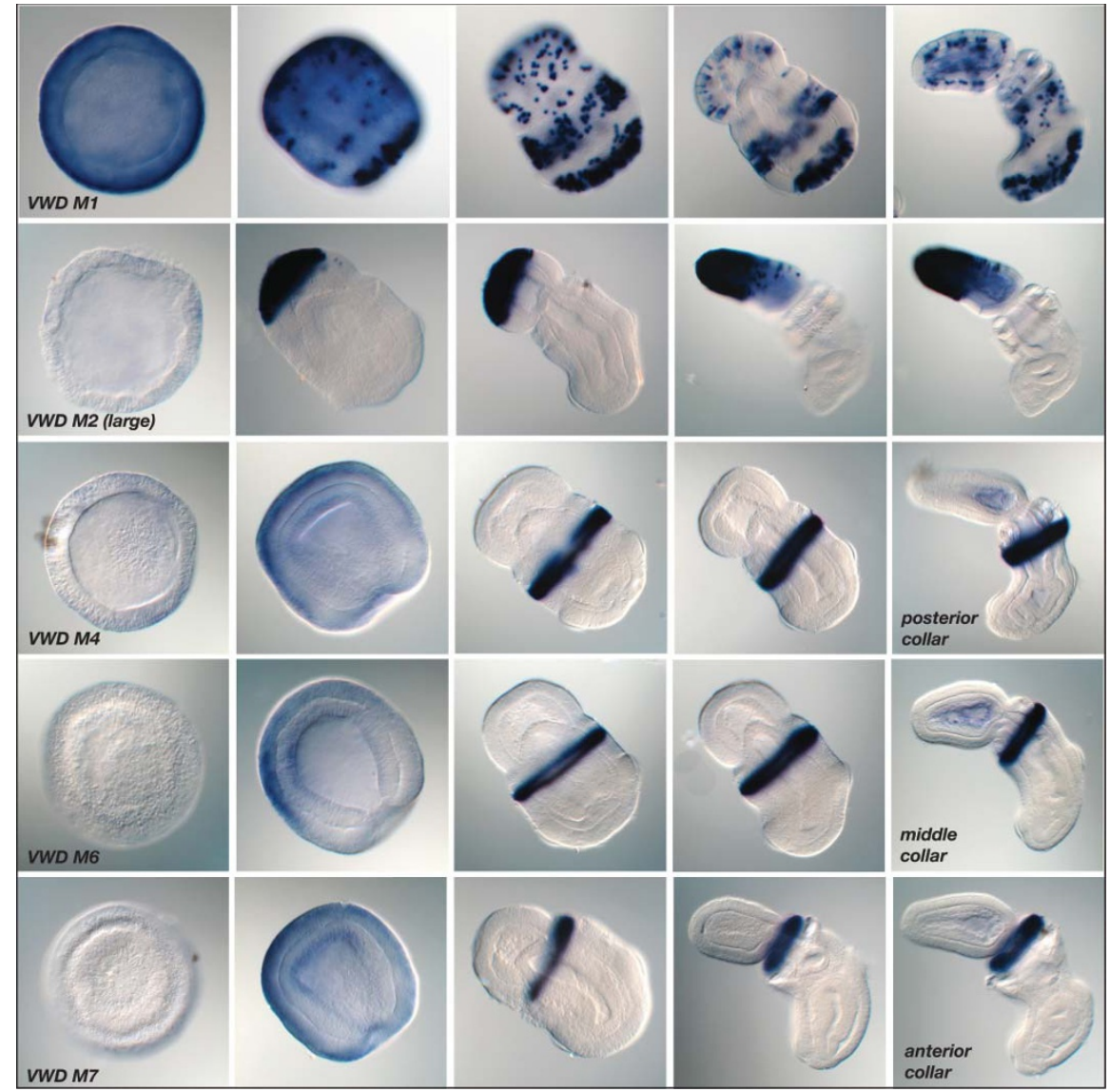

b
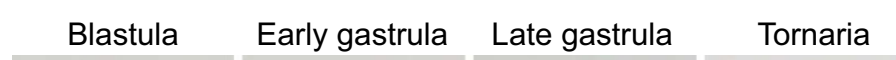

Juvenile
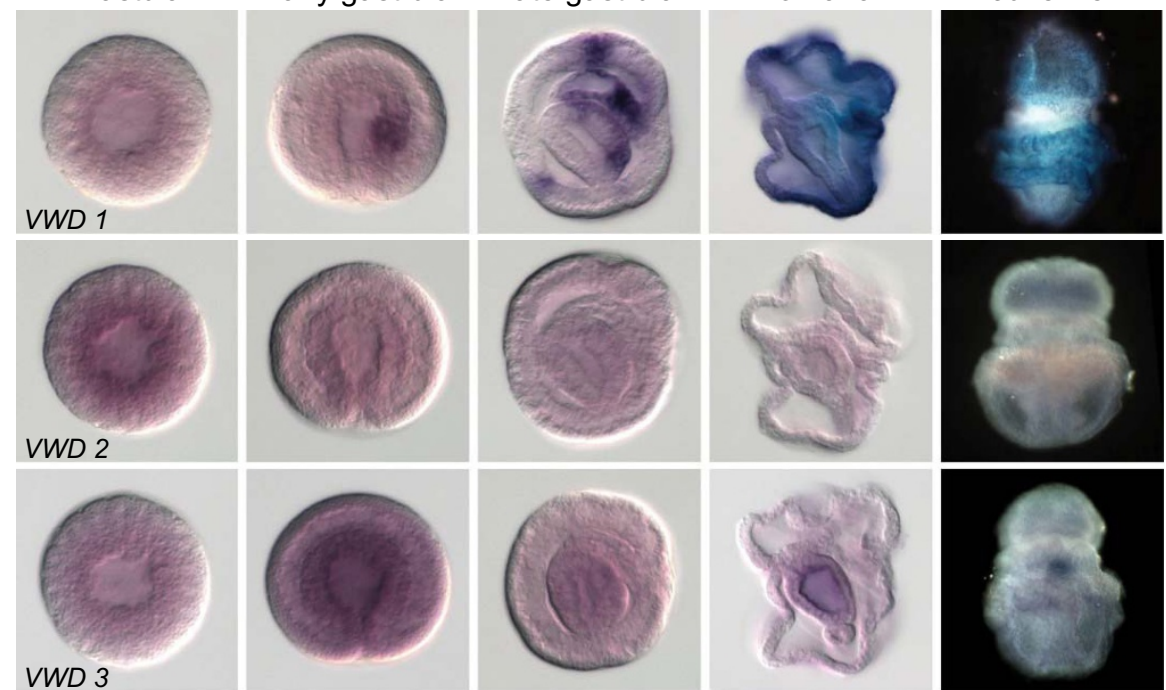

VWD 3
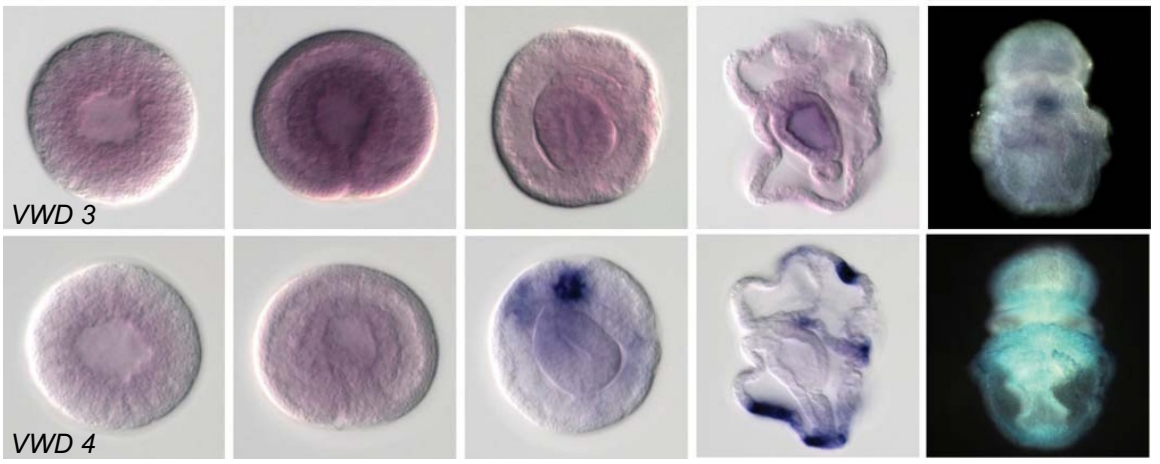

Extended Data Figure 8 | In situ hybridization demonstration of the expression of von Willebrand type D (vWD) domain-encoding genes (putative glycoproteins/mucins) in Saccoglossus and Ptychodera. a, In Saccoglossus the genes are specifically expressed in different

subregions of the ectoderm of the proboscis or collar at these pre-feeding stages. b, In Ptychodera, several of the genes are expressed in endoderm as well as ectoderm of the developing tornaria larva. The sequence IDs for the genes are provided in Supplementary Note S10.4. 
a

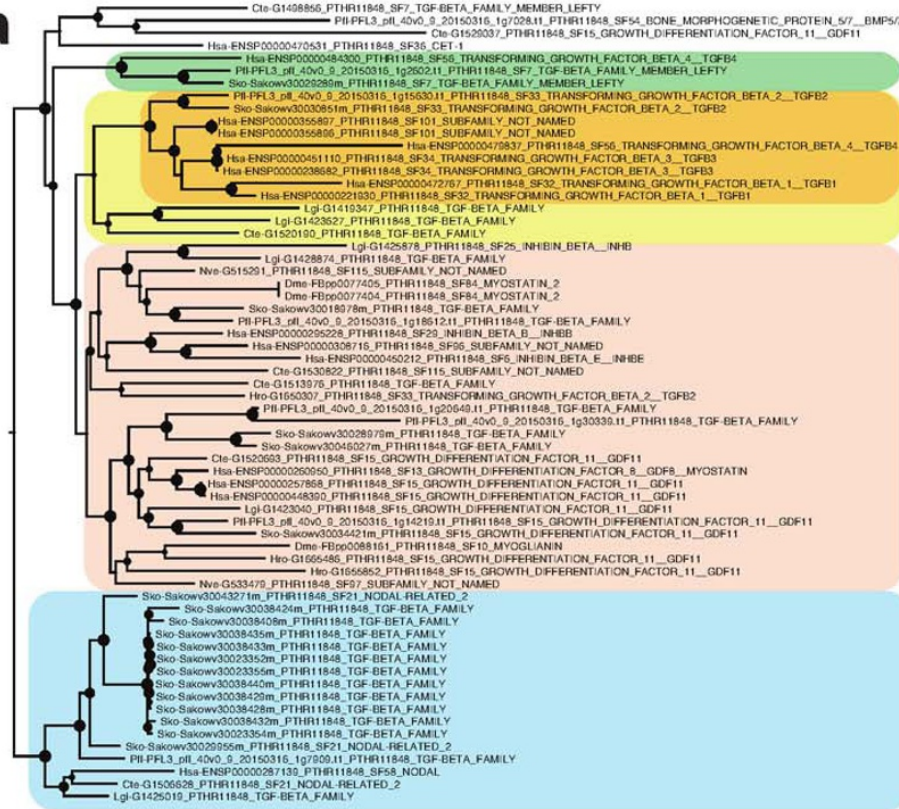

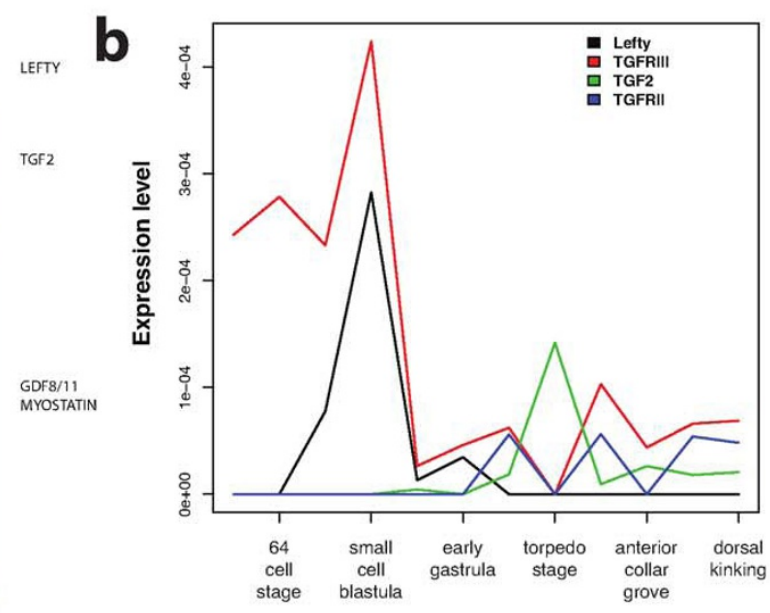

Saccoglossus stages
C
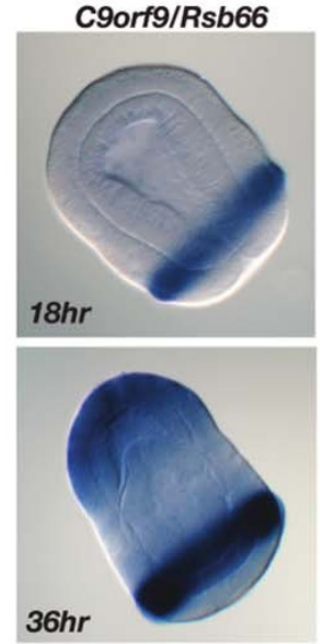

AAADC-like1
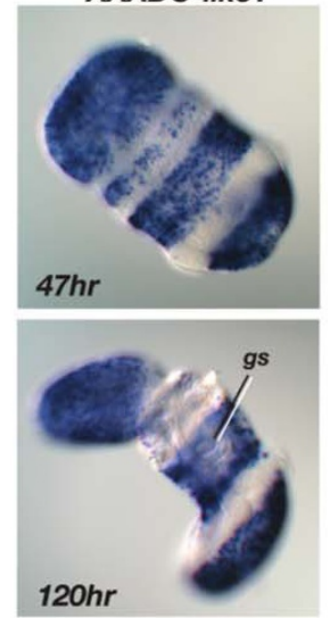

AAADC-like2
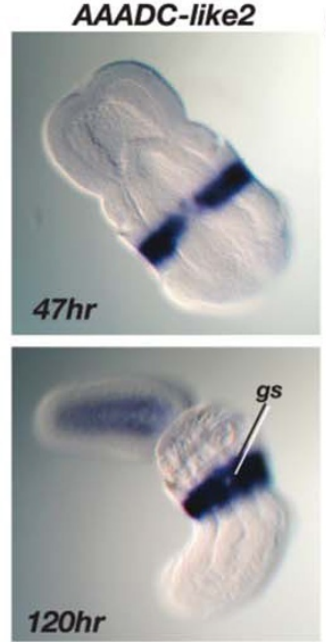

d

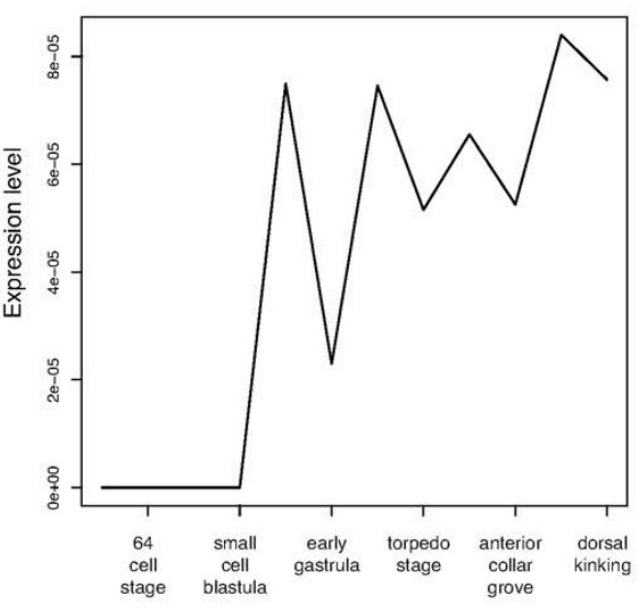

Saccoglossus stages
Extended Data Figure 9 | Gene innovation in deuterostomes. a, FastTree phylogenetic tree of the TGF $\beta$ family members Lefty, TGF 32 , GDF8/11 and Nodal ligands (using GTR model). Bootstrap support is plotted as filled circles (size proportional to the support value) on each node. While Lefty shows deuterostome unique sequence composition, TGF 32 has an acceleration of sequence change at the deuterostome stem branch, compared to the GDF8/11 or Nodal groups. b, Temporal co-expression of Lefty and TGF $\beta$ receptor type III in Saccoglossus at pre-gastrulation developmental stages and of TGF $\beta 2$ and TGF $\beta$ receptor type II at post-gastrulation stages. c, In situ hybridization demonstration of the expression in S. kowalevskii of one of the putative type I novelty genes (c9orf9, also known as rsb66) and of two of AAADC genes (aromatic amino acid decarboxylases of the microbial type) of S. kowalevskii (also in $P$. flava and B. floridae), which closely resemble sequences from bacteria rather than from non-deuterostome metazoans. gs, gill slits. d, The temporal expression profile for $c 9$ orf 9 during $S$. kowalevskii development, taken from transcriptome data. 
Extended Data Table 1 | Examples of deuterostome gene novelties and their genomic features

\begin{tabular}{|c|c|c|c|c|c|c|}
\hline ID & gene family name & $\begin{array}{l}\text { BLAST e-value in non-deuterostome metazoa? } \\
\text { \# or "none" }\end{array}$ & $\begin{array}{l}\text { BLAST e-value in non-metazoan clades with these } \\
\text { domains? } \\
\text { E.g., Ostreocccus, Micromonas, etc. }\end{array}$ & Phylogeny support for novelty/HGT & Origin of novelty? & Putative function in deuterostomes? \\
\hline$\frac{1}{2}$ & $\begin{array}{l}\text { Lefty } \\
\text { Univin, Vg1, DVR, GDF1 }\end{array}$ & $\begin{array}{l}e-12, \text { partial prodomain but no ligand domain in Capitella } \\
\text { weak matches to bmp2/4 sequences }\end{array}$ & & \begin{tabular}{|l} 
EDF9 \\
Figure S10.3.1
\end{tabular} & $\begin{array}{ll}\text { type IV } \\
\text { type IV }\end{array}$ & $\begin{array}{l}\text { antagonist of Nodal signaling. Developmental patterning? } \\
\text { agonist of Nodal signaling. Developmental patterning? }\end{array}$ \\
\hline 3 & TGFb2 & \begin{tabular}{|l|}
$e-35$ for cridarian Aiptasia and sponge Sycon, no protosostmes \\
\end{tabular} & . & EDF9 & type IV & Signaling via Smad2/3 activation. Regulation of cell activities \\
\hline 4 & Thrombospondin $1 / 2$ & $\begin{array}{l}\text { none, except the sponge Amphimedon has a partial } \\
\text { combination of TSP1 and TSP3 domains }\end{array}$ & - & $\begin{array}{l}\text { unique domain combination due to in } \\
\text { TSP1 domains }\end{array}$ & type II & Activation of TGFb2 signals \\
\hline 5 & TGFbR2 & $\begin{array}{l}\text { no matches to de uterostome ectodomain; many matches to } \\
\text { deuterostome protein kinase domain }\end{array}$ & - & Figure $\$ 10.3 .3$ & type IV & Receptor specific for TGFb2 \\
\hline 6 & $\begin{array}{l}\text { UDP-N-acetylglucosamine 2-epimerase/ } \mathrm{N} \text { - } \\
\text { acetylmannosamine kinase }\end{array}$ & $\begin{array}{l}\text { Symsagittifera (acoel) e-154 matching both domains, but not } \\
\text { in Hofstenia (acoel); no protostome match }\end{array}$ & 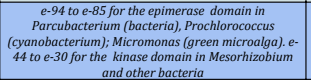 & $\begin{array}{l}\text { No non-deuterostome metazoan sequenes in gene } \\
\text { phylogeny, except acools of uncertain relatedness to } \\
\text { deuterostomes }\end{array}$ & HGT/convergence & $\begin{array}{l}\text { first step of deuterostome sialic acid synthesis; different from } \\
\text { protostome first step }\end{array}$ \\
\hline 7 & $\begin{array}{l}\begin{array}{c}\text { CMP-N-Acetylneuraminic acid hydroxylase } \\
\text { (modifies sialic acid) }\end{array} \\
\end{array}$ & $\begin{array}{l}\text { e-2 for unrelated match in Caenorhabditis brenneri, neither } \\
\text { domain }\end{array}$ & $\begin{array}{l}e-127 \text { to e-86, both domains, ostreococcus and } \\
\text { Micromonas (green microalga) }\end{array}$ & $\begin{array}{c}\text { No non-deuterostome metazoan sequences in gene } \\
\text { phylogeny (S10) }\end{array}$ & HGT/convergence & $\begin{array}{l}\text { produces a glycolyl sialic acid that is also added to } \\
\text { glycocongugates in deuterostomes }\end{array}$ \\
\hline 8 & $\begin{array}{c}\text { alpha-2,6-GalNac-sialyltransferase (expanded } \\
\text { family in Amphioxus and ambulacraria) }\end{array}$ & $\begin{array}{l}\text { e-14 match to ST6Gal sialyltransferases of Tribolium, } \\
\text { Drosophila, and other insects, with idfferent specificity for } \\
\text { oligosaccharide terminus. }\end{array}$ & $\begin{array}{c}\text { e-10 to e-8 for Bathycossus (green microalga) and } \\
\text { Emeliania (coccolithophore) }\end{array}$ & $\begin{array}{l}\text { unclear; deuterostome STGG alNAc connected weakly to } \\
\text { ST6Gal (bilaterian ancestral sequence) }\end{array}$ & type IV & New specificity of sialic linkage compared to protostomes \\
\hline 9 & $\begin{array}{c}\text { alpha-2,3-sialyytransferases (expanded family } \\
\text { in Amphioxus and ambulacraria) }\end{array}$ & \begin{tabular}{|c|} 
e-61 match to Oscarella (sponge) ST3 sequence, and then e-7 \\
match to ST6Gal of insects, with different specificity for \\
oligosacharide terminus
\end{tabular} & $\begin{array}{l}\text { e-17 to e-10 matches to Bathycossus ST3s (green } \\
\text { microalga) and Emeliania (coccolithophore) }\end{array}$ & $\begin{array}{l}\text { unclear; deuterostome ST3 connected to sponge ST3 } \\
\text { and weakly to STGGal (bilaterian ancestral sequence) }\end{array}$ & type IV & New specificity of sialic linkage compared to protostomes \\
\hline 10 & $\begin{array}{l}\text { alpha-2,8-sialyltransferases (greatly } \\
\text { expanded family in Amphioxus and } \\
\text { ambulacraria) }\end{array}$ & $\begin{array}{l}\text { e-7 to ST6Gal of insects, with different specificity for } \\
\text { oligosacharide terminus }\end{array}$ & $\begin{array}{l}\text { e-12 to e-8 matches to Bathy cossus STRs (green } \\
\text { microalga) and Emeliania (coccolithophore) }\end{array}$ & $\begin{array}{l}\text { unclear; deuterostome ST connected weakly to sponge } \\
\text { ST3 and ST6Gal (bilaterian ancestral sequence) }\end{array}$ & type IV & New specificity of sialic linkage compared to protostomes \\
\hline 11 & $\begin{array}{l}\text { B4GGANT, adds N-Actylgal to sialic acid } \\
\text { containing oligosaccharides (expanded family } \\
\text { in Amphioxus and ambulacraria) }\end{array}$ & none & Nitratifractor (epsilon-proteobacterium) & No non-deuterostome metazoans in gene phylogeny & HGT/convergence & synthesis of gangliosides, not found in protostomes \\
\hline 12 & $\begin{array}{l}\text { sialidase 1,2,3,4 (expanded family in } \\
\text { Amphiouxus and ambulacraria) }\end{array}$ & 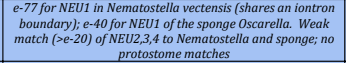 & 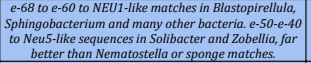 & $\begin{array}{l}\text { No non-deuterostome metazzan sequences in gene } \\
\text { phylogeny (S10) }\end{array}$ & type IV & $\begin{array}{l}\text { removal of sialic acid from novel glycosidic linkages of } \\
\text { deuterostomes }\end{array}$ \\
\hline 13 & 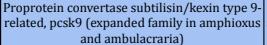 & \begin{tabular}{|l|} 
e-59 match to Platynereis (but no inhibitor domain) and $e-40$ \\
to capitella (different subtilising type), and no intron boundary \\
matches
\end{tabular} & \begin{tabular}{|l|} 
e-103 to e-90 for Kexin-like sequences of \\
Actinobacteria such has SSacharathrix and \\
Saccharomonospora
\end{tabular} & $\begin{array}{l}\text { No non-deuterostome metazoan sequences in gene } \\
\text { phylogeny }\end{array}$ & HGT/convergence & $\begin{array}{c}\text { proteolysis, targets unknown (some signaling receptors in } \\
\text { vertebrates) }\end{array}$ \\
\hline 14 & \begin{tabular}{|c|} 
PAD (Peptidyl Arginyl Deiminase) \\
\end{tabular} & \begin{tabular}{|c|}
$e+1.7$ to an unrelated Ceratitis sequence that lacked PAD \\
domains
\end{tabular} & \begin{tabular}{|c|}
$e-66$ match to Stanieria (cyanobacterium) across all \\
domains
\end{tabular} & $\begin{array}{l}\text { No non-deuterostome metazoan sequences in gene } \\
\text { phylogeny }\end{array}$ & HGT/convergence & $\begin{array}{l}\begin{array}{l}\text { post-translational modification of proteins (change arginyl to } \\
\text { citrullinyl residues) }\end{array} \\
\end{array}$ \\
\hline 15 & $\begin{array}{l}\text { FATSO (Alpha-ketogglutarate-dependent } \\
\text { dioxygenase FTO) }\end{array}$ & & $\begin{array}{l}\text { e-67 to e-60 for matches in Micromonas and } \\
\text { Ostreococcus (green microalgae) }\end{array}$ & $\begin{array}{l}\text { No non-deuterostome metazoan sequences in gene } \\
\text { phylogeny (S10) }\end{array}$ & HGT/convergence & N6-methyladenosine demethylation of nuclear RNAs \\
\hline 16 & Arylsulfatase K-like & 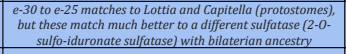 & $\begin{array}{c}\text { e-83 to e-63 for matches of Monosiga } \\
\text { (choanoflagellate) and Arthrobacter (bacterium) }\end{array}$ & $\begin{array}{l}\text { No non-deuterostome metazoan sequences in gene } \\
\text { phylogeny, except distant unrelated sulfatases (S10) }\end{array}$ & HGT/convergence & removal of sulfate groups, targets unknown \\
\hline 17 & polyketide synthase-like & $\begin{array}{l}\text { Metazoan fatty acid synthases e-1 19 toe }-94 \text {, but different } \\
\text { intron-exon structure and domain subtypes. }\end{array}$ & $\begin{array}{c}\text { e=0.0(i.e. <e-200) matches to the bacteria } \\
\text { Flammeovirga (same order of all domains as } \\
\text { deuterostome Family 1), and Sorangium, and the } \\
\text { cyanobacterium Stanieria }\end{array}$ & $\begin{array}{l}\text { No non-deuterostome metazoan sequences in gene } \\
\text { phylogeny }\end{array}$ & HGT/convergence & Synthesis of pigments and antimicrobial agents? \\
\hline 18 & NHL-containing protein & $\begin{array}{l}\text { e-15 matches to protostomian peptidylglycine alpha- } \\
\text { amidating monooxygenases which contains sifferent } \mathrm{NHL} \\
\text { repeats and a copper monoxygenase domain }\end{array}$ & $\begin{array}{l}\text { e-38 matches in planctomycete bacteria such as } \\
\text { Zavarzinella; and in Monosiga (choanoflagellate) }\end{array}$ & $\begin{array}{l}\begin{array}{c}\text { No non-deuterostome metazoan sequences in gene } \\
\text { phylogeny }\end{array} \\
\end{array}$ & HGT/convergence & protein-protein interactions? \\
\hline 19 & choline monoxygenase-like & $>\mathrm{e}-1$ unrelated matches & $\begin{array}{l}\text { e-97 match to Selaginella (spikemosss), e-91 to } \\
\text { Physcomitrella (bryophyte, } \mathrm{e}-90 \text { to Micromonas, } \\
\text { Nannochloropsis, and Coccomyxa (micro-algae) }\end{array}$ & $\begin{array}{l}\text { No non-deuterostome metazoan sequences in gene } \\
\text { phylogeny (S10) }\end{array}$ & HGT/convergence & $\begin{array}{l}\text { first step in the synthesis of betaine, an osmotic protectant and } \\
\text { also a methyl donor for methionine regeneration }\end{array}$ \\
\hline 20 & $\begin{array}{l}\text { ectoine synthase-like (expanded family in } \\
\text { amphioxus and hemichordates) }\end{array}$ & e+7 match unrelated phospholipaseA2 sequence & \begin{tabular}{|c|} 
e-21 to Rhizobium (bacterium \\
(cyanobacteriu
\end{tabular} & $\begin{array}{c}\begin{array}{c}\text { No non-deuterostome metazoan sequences in gene } \\
\text { phylogeny }\end{array} \\
\end{array}$ & HGT/convergence & $\begin{array}{c}\text { a reaction step in the synthesis of ectoine, an osmotic } \\
\text { protectant }\end{array}$ \\
\hline 21 & ectoine hydrolase & $\begin{array}{l}\text { e-18 to e-4 matches to phantoyl dioxygenases of protostomes } \\
\text { and deuterosotmes, containing a distantly related domain }\end{array}$ & $\begin{array}{l}\text { e-69 to e-45 matches to ectoine hydroxylases of high GC } \\
\text { grouup bacteria such as Streptomyces sp. PBH } 53\end{array}$ & No non-deuterosotme sequences in gene phyogeny & HGT/convergence & $\begin{array}{c}\text { a reaction step in the synthesis of betaine, an osmotic } \\
\text { protectant }\end{array}$ \\
\hline 22 & $\begin{array}{l}\text { histidine methyltransferase bacterial-like } \\
\text { (expanded family in anphhiousu and } \\
\text { hemichordates }\end{array}$ & $\begin{array}{c}\text { e-34 to e-7 matches to Crassostrea (oyster) sequences, which } \\
\text { share 2-3 intron boundaries with deuterostome sequences. No } \\
\text { other matches outside deuterostomes }\end{array}$ & $\begin{array}{l}\text { e-46 to e-40 matches to bacteria such as Fimbrimonas, } \\
\text { Kuenenia, and Thiothrix. }\end{array}$ & $\begin{array}{c}\text { Crassostrea sequences more closely associated with } \\
\text { deuterostome sequences on gene phylogeny, than are } \\
\text { bacterial sequences (S10) }\end{array}$ & type IV & Syntheis of trimethyl-histidine derivatives (e.g, ergothionine)? \\
\hline 23 & $\begin{array}{c}\text { Aromatic amino acid decarboxylase microbial } \\
\text { like (expanded family in amphioxus and } \\
\text { hemichordates) }\end{array}$ & $\begin{array}{l}\text { e-46 to e-10 matches to a different kind of aromatic amino } \\
\text { acid decarboxylase in protostomes and deuterostomes, of } \\
\text { bilaterian ancestry. Different intron-exon structure. }\end{array}$ & $\begin{array}{l}\text { e-157 to e-125 matches to Maribacter (marine } \\
\text { bacterium) and other bacteria }\end{array}$ & $\begin{array}{l}\text { No non-deuterostome metazoan sequences in gene } \\
\text { phylogeny (S10) }\end{array}$ & HGT/convergence & $\begin{array}{l}\text { Decarboxylation of aromatic amino acids; neurotransmitter } \\
\text { production? }\end{array}$ \\
\hline 24 & $\begin{array}{l}\text { Cobalamine-independent methionine } \\
\text { synthase }\end{array}$ & $\begin{array}{l}\text { e-115 match to Nematostella (cnidarian) sequence that } \\
\text { sharesan intron boundary with hhe eeutersostome sequences, } \\
\text { indicating bilaterian ancestry. No protostome match }\end{array}$ & $\begin{array}{l}\text { e-119 to e-99 matches to bacteria such as } \\
\text { Planctomycetales and Rhodovibrio }\end{array}$ & $\begin{array}{c}\begin{array}{l}\text { Nematostella sequence more closely associated with } \\
\text { deuterostome sequences on gene phylogeny, than are } \\
\text { bacterial sequences }\end{array} \\
\end{array}$ & type IV & $\begin{array}{l}\text { Addtional pathway of methonine regeneration in } \\
\text { deuterostomes? }\end{array}$ \\
\hline 25 & $\begin{array}{c}\text { Major Facilitator Transporter algal--like, MFS } \\
\text { algal-like }\end{array}$ & $\begin{array}{l}\text { e+1.6 match to unrelated cubilin-like protein of Microplitis } \\
\text { (insect) with no MFS domain }\end{array}$ & $\begin{array}{l}e=0.0(\text { i.e.e }<\text { <-2000) matches to Chlamydimonas, } \\
\text { ostreococcus, Micromonas, and many other micro- } \\
\text { algae }\end{array}$ & $\begin{array}{l}\text { No non-deuterostome metazoan sequences in gene } \\
\text { phylogeny }\end{array}$ & HGT/convergence & Transmembrane export of noxious compounds? \\
\hline 26 & $\begin{array}{l}\text { Multicopper oxidase (MCO); also called } \\
\text { Bilirubinoxidase-lilie (expanded family in } \\
\text { tunicates, amphhioxus and hemichordates) } \\
\text { FaM198-like protein }\end{array}$ & $\begin{array}{c}\text { e-5 matches to insect laccases which contain three insect } \\
\text { cupredoxin domains that differ greatly from bilirubin oxidase } \\
\text { domains }\end{array}$ & \begin{tabular}{|} 
e-75 match to Emeliania (coccolithophore) and $e-65$ to \\
Albugo (oomycete)
\end{tabular} & $\begin{array}{l}\text { No non-deuterostome metazoan sequences in gene } \\
\text { phylogeny }\end{array}$ & HGT/convergence & oxidation of tetrapyrroles? \\
\hline 27 & \begin{tabular}{|c|} 
FAM198-like protein \\
Chromosome 9 Open Reading Frame 9. Rsb66
\end{tabular} & & & $\begin{array}{l}\text { Only deuteroston } \\
\text { Onlydeteroston }\end{array}$ & & unknown \\
\hline$\frac{28}{29}$ & Chromosome 90 pen Reading Frame 9, Rsb66 & $\begin{array}{l}\text { (nemertodermatid) } \\
\text { (-TS }\end{array}$ & . & ydeuteros & Tenetatively type I & unknown, expressed in ciliated cells \\
\hline$\frac{29}{30}$ & n 19-like & $\begin{array}{c}\text { none } \\
\text { none } \\
\end{array}$ & - & $\begin{array}{l}\text { Only deuterosstome sequenes in gene phylogeny } \\
\text { Only deuterostome sequenes in gene phylogeny }\end{array}$ & $\begin{array}{l}\text { type I } \\
\text { type I }\end{array}$ & $\begin{array}{l}\text { unknown } \\
\text { unknown } \\
\end{array}$ \\
\hline 31 & $\begin{array}{c}\begin{array}{c}\text { EF-hand and coiled-coil domain-c } \\
\text { protein 1-like }\end{array} \\
\end{array}$ & none & - & Only deuterostome sequenes in gene phylogeny & type I & unknown \\
\hline
\end{tabular}

This table summarizes the description of the novelties in Supplementary Note 10. 